\title{
Recent development on the geometry of the Teichmüller and moduli spaces of Riemann surfaces
}

\author{
Kefeng Liu, Xiaofeng Sun, and Shing-Tung Yau
}

\section{Contents}

1. Introduction 221

2. The Weil-Petersson metric and its curvature 222

3. The Ricci and perturbed Ricci metrics 227

4. Asymptotics 229

5. Canonical metrics and equivalence 234

6. Goodness of canonical metrics 238

7. Negativity and naturalness 241

8. The Kähler-Ricci flow and Kähler-Einstein metric on the moduli space 244

9. Applications 246

10. Deformation of pluricanonical forms 248

$\begin{array}{ll}\text { References } & 257\end{array}$

\section{Introduction}

The moduli space $\mathcal{M}_{g, k}$ of Riemann surfaces of genus $g$ with $k$ punctures plays an important role in many area of mathematics and theoretical physics. In this article we first survey some of our recent works on the geometry of this moduli space. In the following we assume $g \geq 2$ and $k=0$ to simplify notations. All the results in this paper work for the general case when $3 g-$ $3+k>0$. We will focus on Kähler metrics on the moduli and Teichmüller spaces, especially the Weil-Petersson metric, the Ricci, the perturbed Ricci, and the Kähler-Einstein metrics.

We will review certain new geometric properties we found and proved for these metrics, such as the bounded geometry, the goodness and their naturalness under restriction to boundary divisors. The algebro-geometric 
corollaries such as the stability of the logarithmic cotangent bundles and the infinitesimal rigidity of the moduli spaces will also be briefly discussed. Similar to our previous survey articles $[\mathbf{1 2}, \mathbf{1 1}]$, we will briefly describe the basic ideas of our proofs, the details of the proofs will be published soon, see $[\mathbf{1 3}, \mathbf{1 4}]$.

After introducing the definition of Weil-Petersson metric in Section 2, we discuss the fundamental curvature formula of Wolpert for the Weil-Petersson metric. For the reader's convenience we also describe a proof of the negativity of the Riemannian sectional curvature of the Weil-Petersson metric.

In Section 3 we discuss the Ricci and the perturbed Ricci metrics and their curvature formulas. In Section 4 we describe the asymptotics of these metrics and their curvatures which are important for our understanding of their bounded geometry. In Section 5 we briefly discuss the equivalence of all of the complete metrics on Teichmüller spaces to the Ricci and the perturbed Ricci metrics, which is a simple corollary of our understanding of these two new metrics.

In Section 6 we discuss the goodness of the Weil-Petersson metric, the Ricci, the perturbed Ricci metric and the Kähler-Einstein metric. To prove the goodness we need much more subtle estimates on the connection and the curvatures of these metrics. Section 7 contains discussions of the dual Nakano negativity of the logarithmic tangent bundle of the moduli space and the naturalness of the Ricci and the perturbed Ricci metrics.

In Section 8 we discuss the Kähler-Ricci flow and the Kähler-Einstein metric on the moduli space. There are many interesting corollaries from our understanding of the geometry of the moduli spaces. In Section 9 we discuss the stability of the logarithmic cotangent bundle, the $L^{2}$ cohomology and the infinitesimal rigidity of the moduli spaces as well as the Gauss-Bonnet theorem on the moduli space.

Finally in Section 10 we describe a canonical way to locally deform holomorphic sections the pluricanonical bundles of fibers.

\section{The Weil-Petersson metric and its curvature}

Let $\mathcal{M}_{g}$ be the moduli space of Riemann surfaces of genus $g$ where $g \geq 2$. It is well known that the $\mathcal{M}_{g}$ is a complex orbifold. The Teichmüller space $\mathcal{T}_{g}$, as the space parameterizing marked Riemann surfaces, is a smooth contractible pseudo-convex domain and can be embedded into the Euclidean space of the same dimension.

REMARK 2.1. Since $\mathcal{M}_{g}$ is only an orbifold, in the following when we work near a point $p \in \mathcal{M}_{g}$ which is an orbifold point, we always work on a local manifold cover of $\mathcal{M}_{g}$ around $p$.

An alternative way is to add a level structure on the moduli space so that it becomes smooth [24]. All the following results are still valid. In particular, when we use the universal curve over the moduli space, we always mean the universal curve over the local manifold cover. 
When we deal with global properties of the moduli space, we can use the moduli space with a level structure such that it becomes smooth. We take quotients after we derive the estimates. We can also work on the Teichmüller space which is smooth.

Finally, when we use the universal family over a chart of the moduli space $\mathcal{M}_{g}$, we do not specify any special gauge. All the computations are gauge invariant.

For any point $p \in \mathcal{M}_{g}$ we let $X_{p}$ be the corresponding Riemann surface. By the Kodaira-Spencer theory we have the identification

$$
T_{p}^{1,0} \mathcal{M}_{g} \cong \check{H}^{1}\left(X_{p}, T_{X_{p}}^{1,0}\right) .
$$

It follows from Serre duality that

$$
\Omega_{p}^{1,0} \mathcal{M}_{g} \cong H^{0}\left(X_{p}, K_{X_{p}}^{2}\right)
$$

By the Riemann-Roch theorem we know that the dimension of the moduli space is

$$
\operatorname{dim}_{\mathbb{C}} \mathcal{M}_{g}=n=3 g-3 .
$$

The Weil-Petersson (WP) metric is the first known Kähler metric on $\mathcal{M}_{g}$. Ahlfors showed that the WP metric is Kähler and its holomorphic sectional curvature is bounded from above by a negative constant which only depends on the genus $g$. Royden conjectured that the Ricci curvature of the WP metric is also bounded from above by a negative constant. This conjecture was proved by Wolpert $[\mathbf{2 6}]$.

Now we briefly describe the WP metric and its curvature formula. Please see the works $[\mathbf{3 2}],[\mathbf{3 0}]$ of Wolpert for detailed description and various aspects of the WP metric.

Let $\pi: \mathfrak{X} \rightarrow \mathcal{M}_{g}$ be the universal family over the moduli space. For any point $s \in \mathcal{M}_{g}$ we let $X_{s}=\pi^{-1}(s)$ be the corresponding smooth Riemann surface. Since the Euler characteristic $\chi\left(X_{s}\right)=2-2 g<0$, by the uniformization theorem we know that each fiber $X_{s}$ is equipped with a unique KählerEinstein metric $\lambda$. In the following we will always use the Kähler-Einstein metric $\lambda$ on $X_{s}$. Let $z$ be any holomorphic coordinate on $X_{s}$. We have

$$
\partial_{z} \partial_{\bar{z}} \log \lambda=\lambda \text {. }
$$

Now we fix a point $s \in \mathcal{M}_{g}$ and let $\left(U, s_{1}, \cdots, s_{n}\right)$ be any holomorphic coordinate chart on $\mathcal{M}_{g}$ around $s$. In the following we will denote by $\partial_{i}$ and $\partial_{z}$ the local vector fields $\frac{\partial}{\partial s_{i}}$ and $\frac{\partial}{\partial z}$ respectively. By the Kodaira-Spencer theory and the Hodge theory we have the identification

$$
T_{s}^{1,0} \mathcal{M}_{g} \cong \check{H}^{1}\left(X_{s}, T_{X_{s}}^{1,0}\right) \cong \mathbb{H}^{0,1}\left(X_{s}, T_{X_{s}}^{1,0}\right)
$$


where the right side of the above formula is the space of harmonic Beltrami differentials. In fact we can explicitly construct the above identification. We let

$$
a_{i}=a_{i}(z, s)=-\lambda^{-1} \partial_{i} \partial_{\bar{z}} \log \lambda
$$

and let

$$
v_{i}=\frac{\partial}{\partial s_{i}}+a_{i} \frac{\partial}{\partial z}
$$

The vector field $v_{i}$ is a smooth vector field on $\pi^{-1}(U)$ and is called the harmonic lift of $\frac{\partial}{\partial s_{i}}$. If we let $B_{i}=\bar{\partial}_{F} v_{i} \in A^{0,1}\left(X_{s}, T_{X_{s}}^{1,0}\right)$, then $B_{i}$ is harmonic and the map $\frac{\partial}{\partial s_{i}} \mapsto B_{i}$ is precisely the Kodaira-Spencer map. Here $\bar{\partial}_{F}$ is the operator in the fiber direction. In local coordinates if we let $B_{i}=A_{i} d \bar{z} \otimes \partial_{z}$ then $A_{i}=\partial_{\bar{z}} a_{i}$. Furthermore, it was proved by Schumacher that if $\eta$ is any relative $(1,1)$-form on $\mathfrak{X}$ then

$$
\frac{\partial}{\partial s_{i}} \int_{X_{s}} \eta=\int_{X_{s}} L_{v_{i}} \eta
$$

We note that although $A_{i}$ is a local smooth function on $X_{s}$, the product

$$
A_{i} \bar{A}_{j}=B_{i} \cdot \bar{B}_{j} \in C^{\infty}\left(X_{s}\right)
$$

is globally defined. We let

$$
f_{i \bar{j}}=A_{i} \bar{A}_{j} \in C^{\infty}\left(X_{s}\right)
$$

The Weil-Petersson metric on $\mathcal{M}_{g}$ is given by

$$
h_{i \bar{j}}(s)=\int_{X_{s}} B_{i} \cdot \bar{B}_{j} d v=\int_{X_{s}} f_{i \bar{j}} d v
$$

where $d v=\frac{\sqrt{-1}}{2} \lambda d z \wedge d \bar{z}$ is the volume form on $X_{s}$ with respect to the KählerEinstein metric.

Now we describe the curvature formula of the WP metric. We let

$$
\square=-\lambda^{-1} \partial_{z} \partial_{\bar{z}}
$$

be the Hodge-Laplace operator acting on $C^{\infty}\left(X_{s}\right)$. It is clear that the operator $\square+1$ has no kernel and thus is invertible. We let

$$
e_{i \bar{j}}=(\square+1)^{-1}\left(f_{i \bar{j}}\right) \in C^{\infty}\left(X_{s}\right) .
$$

The following curvature formula is due to Wolpert. See $[\mathbf{9}]$ for the detailed proof.

Proposition 2.1. Let $R_{i \bar{j} k \bar{l}}$ be the curvature of the WP metric. Then

$$
R_{i \bar{j} k \bar{l}}=-\int_{X_{s}}\left(e_{i \bar{j}} f_{k \bar{l}}+e_{i \bar{l}} f_{k \bar{j}}\right) d v
$$


The curvature of the WP metric has very strong negativity property. In fact we shall see in Section 7 that the WP metric is dual Nakano negative. We collect the negativity property of the WP metric in the following proposition.

Proposition 2.2. The bisectional curvature of the WP metric on the moduli space $\mathcal{M}_{g}$ is negative. The holomorphic sectional and Ricci curvatures of the WP metric are bounded from above by negative constants. Furthermore, the Riemannian sectional curvature of the WP metric is also negative.

Proof. These results are well known, see [26]. Here we give a short proof of the negativity of the Riemannian sectional curvature of the WP metric for the reader's convenience. The proof follows from expressing the Riemannian sectional curvature in term of complex curvature tensors and using the curvature formula (2.2).

In general, let $\left(X^{n}, g, J\right)$ be a Kähler manifold. For any point $p \in X$ and two orthonormal real tangent vectors $u, v \in T_{p}^{\mathbb{R}} X$, we let $X=\frac{1}{2}(u-i J u)$ and $Y=\frac{1}{2}(v-i J v)$ and we know that $X, Y \in T_{p}^{1,0} X$. We can choose holomorphic local coordinate $s=\left(s_{1}, \ldots, s_{n}\right)$ around $p$ such that $X=\frac{\partial}{\partial s_{1}}$. If $v=\operatorname{span}_{\mathbb{R}}\{u, J u\}$, since $v$ is orthogonal to $u$ and its length is 1 , we know $v= \pm J u$. In this case we have

$$
R(u, v, u, v)=R(u, J u, u, J u)=4 R_{1 \overline{1} 1 \overline{1}} .
$$

Thus the Riemannian sectional curvature and the holomorphic sectional curvature have the same sign.

If $v$ is not contained in the real plane spanned by $u$ and $J u$ we can choose the coordinate $s$ such that $X=\frac{\partial}{\partial s_{1}}$ and $Y=\frac{\partial}{\partial s_{2}}$. In this case a direct computation shows that

$$
R(u, v, u, v)=2\left(R_{1 \overline{1} 2 \overline{2}}-\operatorname{Re}\left(R_{1 \overline{2}} 1 \overline{2}\right)\right) .
$$

Now we fix a point $p \in \mathcal{M}_{g}$ and let $u, v \in T_{p}^{\mathbb{R}} \mathcal{M}_{g}$. Let $X, Y$ be the corresponding $(1,0)$-vectors. Since we know that the holomorphic sectional curvature of the WP metric is strictly negative, we may assume $v \notin \operatorname{span}_{\mathbb{R}}\{u, J u\}$ and thus we can choose holomorphic local coordinates $s=\left(s_{1}, \ldots, s_{n}\right)$ around $p$ such that $X=\frac{\partial}{\partial s_{1}}(p)$ and $Y=\frac{\partial}{\partial s_{2}}(p)$. By formulas (2.3) and (2.2) we have

$$
\begin{aligned}
R(u, v, u, v) & =-2\left(\int_{X_{p}}\left(e_{1 \overline{1}} f_{2 \overline{2}}+e_{1 \overline{2}} f_{2 \overline{1}}-2 R e\left(e_{1 \overline{2}} f_{1 \overline{2}}\right)\right) d v\right) \\
& =-2\left(\int_{X_{p}}\left(e_{1 \overline{1}} f_{2 \overline{2}}+e_{1 \overline{2}} f_{2 \overline{1}}-e_{1 \overline{2}} f_{1 \overline{2}}-e_{2 \overline{1}} f_{2 \overline{1}}\right) d v\right) .
\end{aligned}
$$

To prove the proposition we only need to show that

$$
\int_{X_{p}} e_{1 \overline{2}} f_{2 \overline{1}} d v \geq \int_{X_{p}} \operatorname{Re}\left(e_{1 \overline{2}} f_{1 \overline{2}}\right) d v
$$


and

$$
\int_{X_{p}} e_{1 \overline{1}} f_{2 \overline{2}} d v \geq \int_{X_{p}} e_{1 \overline{2}} f_{2 \overline{1}} d v
$$

and both equalities cannot hold simultaneously.

To prove inequality $(2.5)$ we let $\alpha=\operatorname{Re}\left(e_{1 \overline{2}}\right)$ and $\beta=\operatorname{Im}\left(e_{1 \overline{2}}\right)$. Then we know

$$
\int_{X_{p}} e_{1 \overline{2}} f_{2 \overline{1}} d v=\int_{X_{p}}(\alpha(\square+1) \alpha+\beta(\square+1) \beta) d v
$$

and

$$
\int_{X_{p}} \operatorname{Re}\left(e_{1 \overline{2}} f_{1 \overline{2}}\right) d v=\int_{X_{p}}(\alpha(\square+1) \alpha-\beta(\square+1) \beta) d v
$$

Thus formula (2.5) reduces to

$$
\int_{X_{p}} \beta(\square+1) \beta d v \geq 0
$$

However, we know

$$
\int_{X_{p}} \beta(\square+1) \beta d v=\int_{X_{p}}\left(\left\|\nabla^{\prime} \beta\right\|^{2}+\beta^{2}\right) d v \geq 0
$$

and the equality holds if and only if $\beta=0$. If this is the case then we know that $e_{1 \overline{2}}$ is a real value function and $f_{1 \overline{2}}$ is real valued too. Since $f_{1 \overline{1}}=A_{1} \bar{A}_{1}$ and $f_{1 \overline{2}}=A_{1} \bar{A}_{2}$ and $f_{1 \overline{1}}$ is real-valued we know that there is a function $f \in C^{\infty}\left(X_{p} \backslash S, \mathbb{R}\right)$ such that $A_{2}=f(z) A_{1}$ on $X_{p} \backslash S$. Here $S$ is the set of zeros of $A_{1}$. Since both $A_{1}$ and $A_{2}$ are harmonic, we know that $\bar{\partial}^{*} A_{1}=\bar{\partial}^{*} A_{2}=0$. These reduce to $\partial_{z}\left(\lambda A_{1}\right)=\partial_{z}\left(\lambda A_{2}\right)=0$ locally. It follows that $\left.\partial_{z} f\right|_{X_{p} \backslash S}=0$. Since $f$ is real-valued we know that $f$ must be a constant. But $A_{1}$ and $A_{2}$ are linearly independent which is a contradiction. So the strict inequality (2.5) always holds.

Now we prove formula (2.6). Let $G(z, w)$ be the Green's function of the operator $\square+1$ and let $T=(\square+1)^{-1}$. By the maximum principle we know that $T$ maps positive functions to positive functions. This implies that the Green's function $G$ is nonnegative. Since $G(z, w)=G(w, z)$ is symmetric we know that

$$
\begin{aligned}
\int_{X_{p}} e_{1 \overline{1}} f_{2 \overline{2}} d v & =\int_{X_{p} \times X_{p}} G(z, w) f_{1 \overline{1}}(w) f_{2 \overline{2}}(z) d v(w) d v(z) \\
& =\frac{1}{2} \int_{X_{p} \times X_{p}} G(z, w)\left(f_{1 \overline{1}}(w) f_{2 \overline{2}}(z)+f_{1 \overline{1}}(z) f_{2 \overline{2}}(w)\right) d v(w) d v(z) .
\end{aligned}
$$


Similarly we have

$$
\begin{aligned}
\int_{X_{p}} e_{1 \overline{2}} f_{2 \overline{1}} d v & =\int_{X_{p} \times X_{p}} G(z, w) f_{1 \overline{2}}(w) f_{2 \overline{1}}(z) d v(w) d v(z) \\
& =\frac{1}{2} \int_{X_{p} \times X_{p}} G(z, w)\left(f_{1 \overline{2}}(w) f_{2 \overline{1}}(z)+f_{1 \overline{2}}(z) f_{2 \overline{1}}(w)\right) d v(w) d v(z) .
\end{aligned}
$$

Formula (2.6) follows from the fact that

$$
\begin{aligned}
& f_{1 \overline{1}}(w) f_{2 \overline{2}}(z)+f_{1 \overline{1}}(z) f_{2 \overline{2}}(w)-f_{1 \overline{2}}(w) f_{2 \overline{1}}(z)-f_{1 \overline{2}}(z) f_{2 \overline{1}}(w) \\
= & \left|A_{1}(z) A_{2}(w)-A_{1}(w) A_{2}(z)\right|^{2} \geq 0 .
\end{aligned}
$$

Although the WP metric has very strong negativity properties, as we shall see in Section 4, the WP metric is not complete and its curvatures have no lower bound.

\section{The Ricci and perturbed Ricci metrics}

In $[\mathbf{9}]$ and $[\mathbf{1 0}]$ we studied two new Kähler metrics: the Ricci metric $\omega_{\tau}$ and the perturbed Ricci metric $\omega_{\widetilde{\tau}}$ on the moduli space $\mathcal{M}_{g}$. These new Kähler metrics are complete and have bounded geometry and thus have many important applications. We now describe these new metrics.

Since the Ricci curvature of the WP metric has negative upper bound, we define the Ricci metric

$$
\omega_{\tau}=-\operatorname{Ric}\left(\omega_{W P}\right) .
$$

We also define the perturbed Ricci metric to be a linear combination of the Ricci metric and the WP metric

$$
\omega_{\widetilde{\tau}}=\omega_{\tau}+C \omega_{W P}
$$

where $C$ is a positive constant. In local coordinates we have $\tau_{i \bar{j}}=-h^{k \bar{l}} R_{i \bar{j} k \bar{l}}$ and $\widetilde{\tau}_{i \bar{j}}=\tau_{i \bar{j}}+C h_{i \bar{j}}$ where $R_{i \bar{j} k \bar{l}}$ is the curvature of the WP metric.

Similar to curvature formula (2.2) of the WP metric we can establish integral formulae for the curvature of the Ricci and perturbed Ricci metrics. These curvature formulae are crucial in estimating the asymptotics of these metrics and their curvature. To establish these formulae, we need to introduce some operators. We let

$$
P: C^{\infty}\left(X_{s}\right) \rightarrow A^{1,0}\left(T_{X_{s}}^{0,1}\right)
$$

be the operator defined by

$$
\left.f \mapsto \partial\left(\omega_{K E}^{-1}\right\lrcorner \partial f\right)
$$


In local coordinate we have $P(f)=\partial_{z}\left(\lambda^{-1} \partial_{z} f\right) d z \otimes \partial_{\bar{z}}$. For each $1 \leq k \leq n$ we let

$$
\xi_{k}: C^{\infty}\left(X_{s}\right) \rightarrow C^{\infty}\left(X_{s}\right)
$$

be the operator defined by

$$
\left.f \mapsto \bar{\partial}^{*}\left(B_{k}\right\lrcorner \partial f\right)=-B_{k} \cdot P(f) .
$$

In the local coordinate we have $\xi_{k}(f)=-\lambda^{-1} \partial_{z}\left(A_{k} \partial_{z} f\right)$. Finally for any $1 \leq k, l \leq n$ we define the operator

$$
Q_{k \bar{l}}: C^{\infty}\left(X_{s}\right) \rightarrow C^{\infty}\left(X_{s}\right)
$$

by

$$
Q_{k \bar{l}}(f)=\bar{P}\left(e_{k \bar{l}}\right) P(f)-2 f_{k \bar{l}} \square f+\lambda^{-1} \partial_{z} f_{k \bar{l}} \partial_{\bar{z}} f .
$$

These operators are commutators of various classical operators on $X_{s}$. See [9] for details. Now we recall the curvature formulae of the Ricci and perturbed Ricci metrics established in [9]. For convenience, we introduce the symmetrization operator.

Definition 3.1. Let $U$ be any quantity which depends on indices $i, k, \alpha$, $\bar{j}, \bar{l}, \bar{\beta}$. The symmetrization operator $\sigma_{1}$ is defined by taking the summation of all orders of the triple $(i, k, \alpha)$. That is

$$
\begin{aligned}
\sigma_{1}(U(i, k, \alpha, \bar{j}, \bar{l}, \bar{\beta}))= & U(i, k, \alpha, \bar{j}, \bar{l}, \bar{\beta})+U(i, \alpha, k, \bar{j}, \bar{l}, \bar{\beta})+U(k, i, \alpha, \bar{j}, \bar{l}, \bar{\beta}) \\
& +U(k, \alpha, i, \bar{j}, \bar{l}, \bar{\beta})+U(\alpha, i, k, \bar{j}, \bar{l}, \bar{\beta})+U(\alpha, k, i, \bar{j}, \bar{l}, \bar{\beta}) .
\end{aligned}
$$

Similarly, $\sigma_{2}$ is the symmetrization operator of $\bar{j}$ and $\bar{\beta}$ and $\widetilde{\sigma_{1}}$ is the symmetrization operator of $\bar{j}, \bar{l}$ and $\bar{\beta}$.

Now we can state the curvature formulae. We let $T=(\square+1)^{-1}$ be the operator in the fiber direction.

TheOREm 3.1. Let $s_{1}, \ldots, s_{n}$ be local holomorphic coordinates at $s \in \mathcal{M}_{g}$ and let $\widetilde{R}_{i \bar{j} k \bar{l}}$ be the curvature of the Ricci metric. Then at $s$, we have

$$
\begin{aligned}
\widetilde{R}_{i \bar{j} k \bar{l}} & =-h^{\alpha \bar{\beta}}\left\{\sigma_{1} \sigma_{2} \int_{X_{s}}\left\{T\left(\xi_{k}\left(e_{i \bar{j}}\right)\right) \bar{\xi}_{l}\left(e_{\alpha \bar{\beta}}\right)+T\left(\xi_{k}\left(e_{i \bar{j}}\right)\right) \bar{\xi}_{\beta}\left(e_{\alpha \bar{l}}\right)\right\} d v\right\} \\
& -h^{\alpha \bar{\beta}}\left\{\sigma_{1} \int_{X_{s}} Q_{k \bar{l}}\left(e_{i \bar{j}}\right) e_{\alpha \bar{\beta}} d v\right\} \\
& \left.+\tau^{p \bar{q}} h^{\alpha \bar{\beta}} h^{\gamma \bar{\delta}}\left\{\sigma_{1} \int_{X_{s}} \xi_{k}\left(e_{i \bar{q}}\right) e_{\alpha \bar{\beta}} d v\right\}\left\{\widetilde{\sigma}_{1} \int_{X_{s}} \bar{\xi}_{l}\left(e_{p \bar{j}}\right) e_{\gamma \bar{\delta}}\right) d v\right\} \\
& +\tau_{p \bar{j}} h^{p \bar{q}} R_{i \bar{q} k \bar{l}} .
\end{aligned}
$$

The curvature formula of the perturbed Ricci metric is similar. 
TheOrem 3.2. Let $\widetilde{\tau}_{i \bar{j}}=\tau_{i \bar{j}}+C h_{i \bar{j}}$ where $\tau$ and $h$ are the Ricci and WP metrics respectively where $C>0$ is a constant. Let $P_{i \bar{j} k \bar{l}}$ be the curvature of the perturbed Ricci metric. Then we have

$$
\begin{aligned}
P_{i \bar{j} k \bar{l}}= & -h^{\alpha \bar{\beta}}\left\{\sigma_{1} \sigma_{2} \int_{X_{s}}\left\{T\left(\xi_{k}\left(e_{i \bar{j}}\right)\right) \bar{\xi}_{l}\left(e_{\alpha \bar{\beta}}\right)+T\left(\xi_{k}\left(e_{i \bar{j}}\right)\right) \bar{\xi}_{\beta}\left(e_{\alpha \bar{l}}\right)\right\} d v\right\} \\
& -h^{\alpha \bar{\beta}}\left\{\sigma_{1} \int_{X_{s}} Q_{k \bar{l}}\left(e_{i \bar{j}}\right) e_{\alpha \bar{\beta}} d v\right\} \\
& \left.+\widetilde{\tau}^{p \bar{q}} h^{\alpha \bar{\beta}} h^{\gamma \bar{\delta}}\left\{\sigma_{1} \int_{X_{s}} \xi_{k}\left(e_{i \bar{q}}\right) e_{\alpha \bar{\beta}} d v\right\}\left\{\widetilde{\sigma}_{1} \int_{X_{s}} \bar{\xi}_{l}\left(e_{p \bar{j}}\right) e_{\gamma \bar{\delta}}\right) d v\right\} \\
& +\tau_{p \bar{j}} h^{p \bar{q}} R_{i \bar{q} k \bar{l}}+C R_{i \bar{j} k \bar{l}} .
\end{aligned}
$$

In $[\mathbf{9}]$ and $[\mathbf{1 0}]$ we proved various properties of these new metrics. Here we collect the important ones.

TheOREM 3.3. The Ricci and perturbed Ricci metrics are complete Kähler metrics on $\mathcal{M}_{g}$. Furthermore we have

- These two metrics have bounded curvature.

- The injectivity radius of the Teichmüller space $\mathcal{T}_{g}$ equipped with any of these two metrics is bounded from below.

- These metrics have Poincaré growth and thus the moduli space has finite volume when equipped with any of these metrics.

- The perturbed Ricci metric has negatively pinched holomorphic sectional and Ricci curvatures when we choose the constant $C$ to be large enough.

The Ricci metric is also cohomologous to the Kähler-Einstein metric on $\mathcal{M}_{g}$ in the sense of currents and hence can be used as the background metric to estimate the Kähler-Einstein metric. We will discuss this in Section 5.

\section{Asymptotics}

Since the moduli space $\mathcal{M}_{g}$ is noncompact, it is important to understand the asymptotic behavior of the canonical metrics in order to study their global properties. We first describe the local pinching coordinates near the boundary of the moduli space by using the plumbing construction of Wolpert.

Let $\mathcal{M}_{g}$ be the moduli space of Riemann surfaces of genus $g \geq 2$ and let $\overline{\mathcal{M}}_{g}$ be its Deligne-Mumford compactification [3]. Each point $y \in \overline{\mathcal{M}}_{g} \backslash \mathcal{M}_{g}$ corresponds to a stable nodal surface $X_{y}$. A point $p \in X_{y}$ is a node if there is a neighborhood of $p$ which is isometric to the germ

$$
\{(u, v)|u v=0,| u|,| v \mid<1\} \subset \mathbb{C}^{2} .
$$


We first recall the rotationally symmetric coordinate (rs-coordinate) on a Riemann surface defined by Wolpert in [28]. There are two cases: the puncture case and the short geodesic case. For the puncture case, we have a nodal surface $X$ and a node $p \in X$. Let $a, b$ be two punctures which are glued together to form $p$.

Definition 4.1. A local coordinate chart $(U, u)$ near $a$ is called rscoordinate if $u(a)=0$ where $u$ maps $U$ to the punctured disc $0<|u|<c$ with $c>0$, and the restriction to $U$ of the Kähler-Einstein metric $\lambda$ on $X$ can be written as

$$
\lambda=\frac{1}{2|u|^{2}(\log |u|)^{2}}|d u|^{2} .
$$

The rs-coordinate $(V, v)$ near $b$ is defined in a similar way.

For the short geodesic case, we have a closed surface $X$, a closed geodesic $\gamma \subset X$ with length $l<c_{*}$ where $c_{*}$ is the collar constant.

Definition 4.2. A local coordinate chart $(U, z)$ is called rs-coordinate at $\gamma$ if $\gamma \subset U$ where $z$ maps $U$ to the annulus $c^{-1}|t|^{\frac{1}{2}}<|z|<c|t|^{\frac{1}{2}}$, and the Kähler-Einstein metric $\lambda$ on $X$ can be written as

$$
\lambda=\frac{1}{2}\left(\frac{\pi}{\log |t|} \frac{1}{|z|} \csc \frac{\pi \log |z|}{\log |t|}\right)^{2}|d z|^{2} .
$$

By Keen's collar theorem [7], we have the following lemma:

LEMMA 4.1. Let $X$ be a closed surface and let $\gamma$ be a closed geodesic on $X$ such that the length $l$ of $\gamma$ satisfies $l<c_{*}$. Then there is a collar $\Omega$ on $X$ with holomorphic coordinate $z$ defined on $\Omega$ such that

(1) $z$ maps $\Omega$ to the annulus $\frac{1}{c} e^{-\frac{2 \pi^{2}}{l}}<|z|<c$ for $c>0$;

(2) the Kähler-Einstein metric on $X$ restricted to $\Omega$ is given by

$$
\left(\frac{1}{2} u^{2} r^{-2} \csc ^{2} \tau\right)|d z|^{2}
$$

where $u=\frac{l}{2 \pi}, r=|z|$ and $\tau=u \log r$;

(3) the geodesic $\gamma$ is given by the equation $|z|=e^{-\frac{\pi^{2}}{l}}$.

We call such a collar $\Omega$ a genuine collar.

We notice that the constant $c$ in the above lemma has a lower bound such that the area of $\Omega$ is bounded from below. Also, the coordinate $z$ in the above lemma is an rs-coordinate. In the following, we will keep the notations $u, r$ and $\tau$.

Now we describe the local manifold cover of $\overline{\mathcal{M}}_{g}$ near the boundary. We take the construction of Wolpert [28]. Let $X_{0,0}$ be a stable nodal surface corresponding to a codimension $m$ boundary point and let $p_{1}, \ldots, p_{m}$ be the nodes of $X_{0.0}$. The smooth part $X_{0}=X_{0,0} \backslash\left\{p_{1}, \ldots, p_{m}\right\}$ is a union of 
punctured Riemann surfaces. We fix rs-coordinate charts $\left(U_{i}, \eta_{i}\right)$ and $\left(V_{i}, \zeta_{i}\right)$ at $p_{i}$ for $i=1, \ldots, m$ such that all the $U_{i}$ and $V_{i}$ are mutually disjoint.

Now we pick an open set $U_{0} \subset X_{0}$ such that the intersection of each connected component of $X_{0}$ and $U_{0}$ is a nonempty relatively compact set and the intersection $U_{0} \cap\left(U_{i} \cup V_{i}\right)$ is empty for all $i$. We pick Beltrami differentials $\nu_{m+1}, \ldots, \nu_{n}$ which are supported in $U_{0}$ and span the tangent space at $X_{0}$ of the deformation space of $X_{0}$. For $s=\left(s_{m+1}, \ldots, s_{n}\right)$, we let $\nu(s)=\sum_{i=m+1}^{n} s_{i} \nu_{i}$. We assume $|s|=\left(\sum\left|s_{i}\right|^{2}\right)^{\frac{1}{2}}$ small enough such that $|\nu(s)|<1$. The nodal surface $X_{0, s}$ is obtained by solving the Beltrami equation $\bar{\partial} w=\nu(s) \partial w$. Since $\nu(s)$ is supported in $U_{0},\left(U_{i}, \eta_{i}\right)$ and $\left(V_{i}, \zeta_{i}\right)$ are still holomorphic coordinates for $X_{0, s}$. We note that these coordinates are no longer rs-coordinates with respect to the complex structure on $X_{0, s}$. By the theory of Ahlfors and Bers [1] and Wolpert [28] we can assume that there are constants $\delta, c>0$ such that when $|s|<\delta, \eta_{i}$ and $\zeta_{i}$ are holomorphic coordinates on $X_{0, s}$ with $0<\left|\eta_{i}\right|<c$ and $0<\left|\zeta_{i}\right|<c$.

Now we assume $t=\left(t_{1}, \ldots, t_{m}\right)$ has small norm. We do the plumbing construction on $X_{0, s}$ to obtain $X_{t, s}$ in the following way. We remove from $X_{0, s}$ the discs $0<\left|\eta_{i}\right| \leq \frac{\left|t_{i}\right|}{c}$ and $0<\left|\zeta_{i}\right| \leq \frac{\left|t_{i}\right|}{c}$ for each $i=1, \ldots, m$, and identify $\frac{\left|t_{i}\right|}{c}<\left|\eta_{i}\right|<c$ with $\frac{\left|t_{i}\right|}{c}<\left|\zeta_{i}\right|<c$ by the rule $\eta_{i} \zeta_{i}=t_{i}$. This defines the surface $X_{t, s}$. The tuple $\left(t_{1}, \ldots, t_{m}, s_{m+1}, \ldots, s_{n}\right)$ are the local pinching coordinates for the manifold cover of $\overline{\mathcal{M}}_{g}$. We call the coordinates $\eta_{i}$ (or $\zeta_{i}$ ) the plumbing coordinates on $X_{t, s}$ and the collar defined by $\frac{\left|t_{i}\right|}{c}<\left|\eta_{i}\right|<c$ the plumbing collar.

REMARK 4.1. From the estimate of Wolpert [27], [28] on the length of short geodesic, we have $u_{i}=\frac{l_{i}}{2 \pi} \sim-\frac{\pi}{\log \left|t_{i}\right|}$.

Let $(t, s)=\left(t_{1}, \ldots, t_{m}, s_{m+1}, \ldots, s_{n}\right)$ be the pinching coordinates near $X_{0,0}$. For $|(t, s)|<\delta$, let $\Omega_{c}^{j}$ be the $j$-th genuine collar on $X_{t, s}$ which contains a short geodesic $\gamma_{j}$ with length $l_{j}$.

In the rest of this section we will use the following notations:

$$
\left\{\begin{array}{l}
u_{j}=\frac{l_{j}}{2 \pi} \\
u_{0}=\sum_{j=1}^{m} u_{j}+\sum_{j=m+1}^{n}\left|s_{j}\right| \\
r_{j}=\left|z_{j}\right| \\
\tau_{j}=u_{j} \log r_{j}
\end{array}\right.
$$

where $z_{j}$ is the properly normalized rs-coordinate on $\Omega_{c}^{j}$ such that

$$
\Omega_{c}^{j}=\left\{z_{j}\left|c^{-1} e^{-\frac{2 \pi^{2}}{l_{j}}}<\right| z_{j} \mid<c\right\}
$$


From the above argument, we know that the Kähler-Einstein metric $\lambda$ on $X_{t, s}$, restrict to the collar $\Omega_{c}^{j}$, is given by

$$
\lambda=\frac{1}{2} u_{j}^{2} r_{j}^{-2} \csc ^{2} \tau_{j} .
$$

For convenience, we let $\Omega_{c}=\cup_{j=1}^{m} \Omega_{c}^{j}$ and $R_{c}=X_{t, s} \backslash \Omega_{c}$. In the following, we may change the constant $c$ finitely many times. It is clear that this will not affect the estimates.

To estimate the WP, Ricci and perturbed Ricci metrics and their curvatures, we first need to to find all the harmonic Beltrami differentials $B_{1}, \ldots, B_{n}$ corresponding to the tangent vectors $\frac{\partial}{\partial t_{1}}, \ldots, \frac{\partial}{\partial s_{n}}$. In [17], Masur constructed $3 g-3$ regular holomorphic quadratic differentials $\psi_{1}, \ldots, \psi_{n}$ on the plumbing collars by using the plumbing coordinate $\eta_{j}$. These quadratic differentials correspond to the cotangent vectors $d t_{1}, \ldots, d s_{n}$.

However, it is more convenient to estimate the curvature if we use the rscoordinate on $X_{t, s}$ since we have the accurate form of the Kähler-Einstein metric $\lambda$ in this coordinate. In [25], Trapani used the graft metric constructed by Wolpert [28] to estimate the difference between the plumbing coordinate and rs-coordinate. He also described the holomorphic quadratic differentials constructed by Masur in the rs-coordinate. We collect Trapani's results (Lemma $6.2-6.5,[\mathbf{2 5}]$ ) in the following theorem:

TheOREM 4.1. Let $(t, s)$ be the pinching coordinates on $\overline{\mathcal{M}}_{g}$ near $X_{0,0}$ which corresponds to a codimension $m$ boundary point of $\overline{\mathcal{M}}_{g}$. Then there exist constants $M, \delta>0$ and $1>c>0$ such that if $|(t, s)|<\delta$, then the $j$-th plumbing collar on $X_{t, s}$ contains the genuine collar $\Omega_{c}^{j}$. Furthermore, one can choose rs-coordinate $z_{j}$ on the collar $\Omega_{c}^{j}$ such that the holomorphic quadratic differentials $\psi_{1}, \ldots, \psi_{n}$ corresponding to the cotangent vectors $d t_{1}, \ldots, d s_{n}$ have the form $\psi_{i}=\varphi_{i}\left(z_{j}\right) d z_{j}^{2}$ on the genuine collar $\Omega_{c}^{j}$ for $1 \leq j \leq m$, where

$$
\begin{aligned}
& \text { (1) } \varphi_{i}\left(z_{j}\right)=\frac{1}{z_{j}^{2}}\left(q_{i}^{j}\left(z_{j}\right)+\beta_{i}^{j}\right) \text { if } i \geq m+1 ; \\
& \text { (2) } \varphi_{i}\left(z_{j}\right)=\left(-\frac{t_{j}}{\pi}\right) \frac{1}{z_{j}^{2}}\left(q_{j}\left(z_{j}\right)+\beta_{j}\right) \text { if } i=j ; \\
& \text { (3) } \varphi_{i}\left(z_{j}\right)=\left(-\frac{t_{i}}{\pi}\right) \frac{1}{z_{j}^{2}}\left(q_{i}^{j}\left(z_{j}\right)+\beta_{i}^{j}\right) \text { if } 1 \leq i \leq m \text { and } i \neq j .
\end{aligned}
$$

Here $\beta_{i}^{j}$ and $\beta_{j}$ are functions of $(t, s), q_{i}^{j}$ and $q_{j}$ are functions of $\left(t, s, z_{j}\right)$ given by

$$
q_{i}^{j}\left(z_{j}\right)=\sum_{k<0} \alpha_{i k}^{j}(t, s) t_{j}^{-k} z_{j}^{k}+\sum_{k>0} \alpha_{i k}^{j}(t, s) z_{j}^{k}
$$

and

$$
q_{j}\left(z_{j}\right)=\sum_{k<0} \alpha_{j k}(t, s) t_{j}^{-k} z_{j}^{k}+\sum_{k>0} \alpha_{j k}(t, s) z_{j}^{k}
$$

such that

(1) $\sum_{k<0}\left|\alpha_{i k}^{j}\right| c^{-k} \leq M$ and $\sum_{k>0}\left|\alpha_{i k}^{j}\right| c^{k} \leq M$ if $i \neq j$;

(2) $\sum_{k<0}\left|\alpha_{j k}\right| c^{-k} \leq M$ and $\sum_{k>0}\left|\alpha_{j k}\right| c^{k} \leq M$; 
(3) $\left|\beta_{i}^{j}\right|=O\left(\left|t_{j}\right|^{\frac{1}{2}-\epsilon}\right)$ with $\epsilon<\frac{1}{2}$ if $i \neq j$;

(4) $\left|\beta_{j}\right|=\left(1+O\left(u_{0}\right)\right)$.

An immediate consequence is the precise asymptotics of the WP metric which was computed in $[\mathbf{9}]$. These asymptotic estimates were also given by Wolpert in $[\mathbf{2 9}]$.

THEOREM 4.2. Let $(t, s)$ be the pinching coordinates and let $h$ be the WP metric. Then

(1) $h^{i \bar{i}}=2 u_{i}^{-3}\left|t_{i}\right|^{2}\left(1+O\left(u_{0}\right)\right)$ and $h_{i \bar{i}}=\frac{1}{2} \frac{u_{i}^{3}}{\left|t_{i}\right|^{2}}\left(1+O\left(u_{0}\right)\right)$ for $1 \leq i \leq m$;

(2) $h^{i \bar{j}}=O\left(\left|t_{i} t_{j}\right|\right)$ and $h_{i \bar{j}}=O\left(\frac{u_{i}^{3} u_{j}^{3}}{\left|t_{i} t_{j}\right|}\right)$, if $1 \leq i, j \leq m$ and $i \neq j$;

(3) $h^{i \bar{j}}=O(1)$ and $h_{i \bar{j}}=O(1)$, if $m+1 \leq i, j \leq n$;

(4) $h^{i \bar{j}}=O\left(\left|t_{i}\right|\right)$ and $h_{i \bar{j}}=O\left(\frac{u_{i}^{3}}{\left|t_{i}\right|}\right)$ if $i \leq m<j$;

(5) $h^{i \bar{j}}=O\left(\left|t_{j}\right|\right)$ and $h_{i \bar{j}}=O\left(\frac{u_{j}^{3}}{\left|t_{j}\right|}\right)$ if $j \leq m<i$.

By using the asymptotics of the WP metric and the fact that

$$
B_{i}=\lambda^{-1} \sum_{j=1}^{n} h_{i j} \bar{\psi}_{j}
$$

we can derive the expansion of the harmonic Beltrami differentials corresponding to $\frac{\partial}{\partial t_{i}}$ and $\frac{\partial}{\partial s_{j}}$.

THEOREM 4.3. For c small, on the genuine collar $\Omega_{c}^{j}$, the coefficient functions $A_{i}$ of the harmonic Beltrami differentials have the form:

(1) $A_{i}=\frac{z_{j}}{z_{j}} \sin ^{2} \tau_{j}\left(\overline{p_{i}^{j}\left(z_{j}\right)}+\overline{b_{i}^{j}}\right)$ if $i \neq j$;

(2) $A_{j}=\frac{z_{j}}{z_{j}} \sin ^{2} \tau_{j}\left(\overline{p_{j}\left(z_{j}\right)}+\overline{b_{j}}\right)$

where

(1) $p_{i}^{j}\left(z_{j}\right)=\sum_{k \leq-1} a_{i k}^{j} \rho_{j}^{-k} z_{j}^{k}+\sum_{k \geq 1} a_{i k}^{j} z_{j}^{k}$ if $i \neq j$;

(2) $p_{j}\left(z_{j}\right)=\sum_{k \leq-1} a_{j k} \rho_{j}^{-k} z_{j}^{k}+\sum_{k \geq 1} a_{j k} z_{j}^{k}$.

In the above expressions, $\rho_{j}=e^{-\frac{2 \pi^{2}}{l_{j}}}$ and the coefficients satisfy the following conditions:

(1) $\sum_{k \leq-1}\left|a_{i k}^{j}\right| c^{-k}=O\left(u_{j}^{-2}\right)$ and $\sum_{k \geq 1}\left|a_{i k}^{j}\right| c^{k}=O\left(u_{j}^{-2}\right)$ if $i \geq m+1$;

(2) $\sum_{k \leq-1}\left|a_{i k}^{j}\right| c^{-k}=O\left(\frac{u_{i}^{3} u_{j}^{-2}}{\left|t_{i}\right|}\right)$ and $\sum_{k \geq 1}\left|a_{i k}^{j}\right| c^{k}=O\left(\frac{u_{i}^{3} u_{j}^{-2}}{\left|t_{i}\right|}\right)$ if $i \leq m$ and $i \neq j$;

(3) $\sum_{k \leq-1}\left|a_{j k}\right| c^{-k}=O\left(\frac{u_{j}}{\left|t_{j}\right|}\right)$ and $\sum_{k \geq 1}\left|a_{j k}\right| c^{k}=O\left(\frac{u_{j}}{\left|t_{j}\right|}\right)$; 
(4) $\left|b_{i}^{j}\right|=O\left(u_{j}\right)$ if $i \geq m+1$;

(5) $\left|b_{i}^{j}\right|=O\left(u_{j}\right) O\left(\frac{u_{i}^{3}}{\left|t_{i}\right|}\right)$ if $i \leq m$ and $i \neq j$;

(6) $b_{j}=-\frac{u_{j}}{\pi \overline{t_{j}}}\left(1+O\left(u_{0}\right)\right)$.

By a detailed study of the curvature of the WP metric we derived the precise asymptotics of the Ricci metric in $[\mathbf{9}]$.

TheOREM 4.4. Let $(t, s)$ be the pinching coordinates. Then we have

(1) $\tau_{i \bar{i}}=\frac{3}{4 \pi^{2}} \frac{u_{i}^{2}}{\left|t_{i}\right|^{2}}\left(1+O\left(u_{0}\right)\right)$ and $\tau^{i \bar{i}}=\frac{4 \pi^{2}}{3} \frac{\left|t_{i}\right|^{2}}{u_{i}^{2}}\left(1+O\left(u_{0}\right)\right)$, if $i \leq m$;

(2) $\tau_{i \bar{j}}=O\left(\frac{u_{i}^{2} u_{j}^{2}}{\left|t_{i} t_{j}\right|}\left(u_{i}+u_{j}\right)\right)$ and $\tau^{i \bar{j}}=O\left(\left|t_{i} t_{j}\right|\right)$, if $i, j \leq m$ and $i \neq j$;

(3) $\tau_{i \bar{j}}=O\left(\frac{u_{i}^{2}}{\left|t_{i}\right|}\right)$ and $\tau^{i \bar{j}}=O\left(\left|t_{i}\right|\right)$, if $i \leq m$ and $j \geq m+1$;

(4) $\tau_{i \bar{j}}=O\left(\frac{u_{j}^{2}}{\left|t_{j}\right|}\right)$ and $\tau^{i \bar{j}}=O\left(\left|t_{j}\right|\right)$, if $j \leq m$ and $i \geq m+1$;

(5) $\tau_{i \bar{j}}=O(1)$, if $i, j \geq m+1$.

In $[\mathbf{9}]$ we also derived the asymptotics of the curvature of the Ricci metric.

TheOREM 4.5. Let $X_{0} \in \overline{\mathcal{M}_{g}} \backslash \mathcal{M}_{g}$ be a codimension $m$ point and let $\left(t_{1}, \ldots, t_{m}, s_{m+1}, \ldots, s_{n}\right)$ be the pinching coordinates at $X_{0}$ where $t_{1}, \ldots, t_{m}$ correspond to the degeneration directions. Then the holomorphic sectional curvature is negative in the degeneration directions and is bounded in the non-degeneration directions. More precisely, there exists $\delta>0$ such that, if $|(t, s)|<\delta$, then

$$
\widetilde{R}_{i \bar{i} i \bar{i}}=-\frac{3 u_{i}^{4}}{8 \pi^{4}\left|t_{i}\right|^{4}}\left(1+O\left(u_{0}\right)\right)
$$

if $i \leq m$ and

$$
\left|\widetilde{R}_{i \bar{i} i \bar{i}}\right|=O(1)
$$

if $i \geq m+1$. Here $\widetilde{R}$ is the curvature of the Ricci metric.

Furthermore, on $\mathcal{M}_{g}$, the holomorphic sectional curvature, the bisectional curvature and the Ricci curvature of the Ricci metric are bounded from above and below.

In $[\mathbf{1 3}]$ and [14] we derived more precise estimates of the curvature of the Ricci and perturbed Ricci metrics which we will discuss in Section 6.

\section{Canonical metrics and equivalence}

In addition to the WP, Ricci and perturbed Ricci metrics on the moduli space, there are several other canonical metrics on $\mathcal{M}_{g}$. These include the 
Teichmüller metric, the Kobayashi metric, the Carathéodory metric, the Kähler-Einstein metric, the induced Bergman metric, the McMullen metric and the asymptotic Poincaré metric.

Firstly, on any complex manifold there are two famous Finsler metrics: the Carathéodory and Kobayashi metrics. Now we describe these metrics.

Let $X$ be a complex manifold and of dimension $n$. let $\Delta_{R}$ be the disk in $\mathbb{C}$ with radius $R$. Let $\Delta=\Delta_{1}$ and let $\rho$ be the Poincaré metric on $\Delta$. Let $p \in X$ be a point and let $v \in T_{p} X$ be a holomorphic tangent vector. Let $\operatorname{Hol}\left(X, \Delta_{R}\right)$ and $\operatorname{Hol}\left(\Delta_{R}, X\right)$ be the spaces of holomorphic maps from $X$ to $\Delta_{R}$ and from $\Delta_{R}$ to $X$ respectively. The Carathéodory norm of the vector $v$ is defined to be

$$
\|v\|_{C}=\sup _{f \in \operatorname{Hol}(X, \Delta)}\left\|f_{*} v\right\|_{\Delta, \rho}
$$

and the Kobayashi norm of $v$ is defined to be

$$
\|v\|_{K}=\in f_{f \in \operatorname{Hol}\left(\Delta_{R}, X\right), f(0)=p, f^{\prime}(0)=v} \frac{2}{R} .
$$

It is well known that the Carathéodory metric is bounded from above by the Kobayashi metric after proper normalization.

The first known metric on the Teichmüller space $\mathcal{T}_{g}$ is the Teichmüller metric which is also an Finsler metric. Royden showed that, on $\mathcal{T}_{g}$, the Teichmüller metric coincides with the Kobayashi metric. Generalizations and proofs of Royden's theorem can be found in [16].

Now we look at the Kähler metrics. Firstly, since the Teichmüller space $\mathcal{T}_{g}$ is a pseudo-convex domain, by the work of Cheng and Yau [2] and the later work of Yau, there exist a unique complete Kähler-Einstein metric on $\mathcal{T}_{g}$ whose Ricci curvature is -1 .

There is also a canonical Bergman metric on $\mathcal{T}_{g}$ which we describe now. In general, let $X$ be any complex manifold, let $K_{X}$ be the canonical bundle of $X$ and let $W$ be the space of $L^{2}$ holomorphic sections of $K_{X}$ in the sense that if $\sigma \in W$, then

$$
\|\sigma\|_{L^{2}}^{2}=\int_{X}(\sqrt{-1})^{n^{2}} \sigma \wedge \bar{\sigma}<\infty .
$$

The inner product on $W$ is defined to be

$$
(\sigma, \rho)=\int_{X}(\sqrt{-1})^{n^{2}} \sigma \wedge \bar{\rho}
$$

for all $\sigma, \rho \in W$. Let $\sigma_{1}, \sigma_{2}, \ldots$ be an orthonormal basis of $W$. The Bergman kernel form is the non-negative $(n, n)$-form

$$
B_{X}=\sum_{j=1}^{\infty}(\sqrt{-1})^{n^{2}} \sigma_{j} \wedge \bar{\sigma}_{j} .
$$


With a choice of local coordinates $z_{i}, \ldots, z_{n}$, we have

$$
B_{X}=B E_{X}(z, \bar{z})(\sqrt{-1})^{n^{2}} d z_{1} \wedge \cdots \wedge d z_{n} \wedge d \bar{z}_{1} \wedge \cdots \wedge d \bar{z}_{n}
$$

where $B E_{X}(z, \bar{z})$ is called the Bergman kernel function. If the Bergman kernel $B_{X}$ is positive, one can define the Bergman metric

$$
B_{i \bar{j}}=\frac{\partial^{2} \log B E_{X}(z, \bar{z})}{\partial z_{i} \partial \bar{z}_{j}} .
$$

The Bergman metric is well-defined and is nondegenerate if the elements in $W$ separate points and the first jet of $X$.

It is easy to see that both the Kähler-Einstein metric and the Bergman metric on the Teichmüller space $\mathcal{T}_{g}$ are invariant under the action of the mapping class group and thus descend down to the moduli space.

REMARK 5.1. We note that the induced Bergman metric on $\mathcal{M}_{g}$ is different from the Bergman metric on $\mathcal{M}_{g}$.

In [18] McMullen introduced another Kähler metric $g_{1 / l}$ on $\mathcal{M}_{g}$ which is equivalent to the Teichmüller metric. Let $\log : \mathbb{R}_{+} \rightarrow[0, \infty)$ be a smooth function such that

(1) $\log (x)=\log x$ if $x \geq 2$;

(2) $\log (x)=0$ if $x \leq 1$.

For suitable choices of small constants $\delta, \epsilon>0$, the Kähler form of the McMullen metric $g_{1 / l}$ is

$$
\omega_{1 / l}=\omega_{W P}-i \delta \sum_{l_{\gamma}(X)<\epsilon} \partial \bar{\partial} \log \frac{\epsilon}{l_{\gamma}}
$$

where the sum is taken over primitive short geodesics $\gamma$ on $X$.

Finally, since $\mathcal{M}_{g}$ is quasi-projective, there exists a non-canonical asymptotic Poincaré metric $\omega_{P}$ on $\mathcal{M}_{g}$.

In general, Let $\bar{M}$ be a compact projective manifold of dimension $m$. Let $Y \subset \bar{M}$ be a divisor of normal crossings and let $M=\bar{M} \backslash Y$. We cover $\bar{M}$ by coordinate charts $U_{1}, \ldots, U_{p}, \ldots, U_{q}$ such that $\left(\bar{U}_{p+1} \cup \cdots \cup \bar{U}_{q}\right) \cap Y=\emptyset$. We also assume that, for each $1 \leq \alpha \leq p$, there is a constant $n_{\alpha}$ such that $U_{\alpha} \backslash Y=\left(\Delta^{*}\right)^{n_{\alpha}} \times \Delta^{m-n_{\alpha}}$ and on $U_{\alpha}, Y$ is given by $z_{1}^{\alpha} \ldots z_{n_{\alpha}}^{\alpha}=0$. Here $\Delta$ is the disk of radius $\frac{1}{2}$ and $\Delta^{*}$ is the punctured disk of radius $\frac{1}{2}$. Let $\left\{\eta_{i}\right\}_{1 \leq i \leq q}$ be a partition of unity subordinate to the cover $\left\{U_{i}\right\}_{1 \leq i \leq q}$. Let $\omega$ be a Kähler metric on $\bar{M}$ and let $C$ be a positive constant. Then for $C$ large, the Kähler form

$$
\omega_{P}=C \omega+\sum_{i=1}^{p} \sqrt{-1} \partial \bar{\partial}\left(\eta_{i} \log \log \frac{1}{\left|z_{1}^{i} \cdots z_{n_{i}}^{i}\right|}\right)
$$

defines a complete metric on $M$ with finite volume. This is because on each $U_{i}$ with $1 \leq i \leq p, \omega_{p}$ is bounded from above and below by the local Poincaré metric on $U_{i}$. We call this metric the asymptotic Poincaré metric. 
In 2004, we proved in [9] that all complete metrics on the moduli space are equivalent. The proof is based on asymptotic analysis of these metrics and Yau's Schwarz Lemma. The second author showed our result to several geometers in April of 2004. We are grateful to Professor S.K. Yeung for showing great interest.

In July of the same year, we learnt from the abstract of a seminar announcement in Hong Kong university that Yeung was presenting a proof of our result using a new idea of bounded pluri-subharmonic functions. However, by November, Yeung, in a printed paper, returned to use our argument to prove our result. It is curious to know whether the idea of bounded pluri-subharmonic functions can be used.

We recall that two metrics on $\mathcal{M}_{g}$ are equivalent if one metric is bounded from above and below by positive constant multiples of the other metric.

THEOREM 5.1. On the moduli space $\mathcal{M}_{g}$ the Ricci metric, the perturbed Ricci metric, the Kähler-Einstein metric, the induced Bergman metric, the McMullen metric, the asymptotic Poincaré metric, the Carathéodory metric and the Teichmüller-Kobayashi metric are equivalent.

The equivalence of several of these metrics hold in more general setting. In 2004 we defined the holomorphic homogeneous regular manifolds in [9] which generalized the idea of Morrey.

Definition 5.1. A complex manifold $X$ of dimension $n$ is called holomorphic homogeneous regular if there are positive constants $r<R$ such that for each point $p \in X$ there is a holomorphic map $f_{p}: X \rightarrow \mathbb{C}^{n}$ which satisfies

(1) $f_{p}(p)=0$;

(2) $f_{p}: X \rightarrow f_{p}(X)$ is a biholomorphism;

(3) $B_{r} \subset f_{p}(X) \subset B_{R}$ where $B_{r}$ and $B_{R}$ are Euclidean balls with center 0 in $\mathbb{C}^{n}$.

In 2009, Yeung [33] basically reproduced our definition, but with a different name: domain of uniform squeezing property. In any case, the restriction properties of canonical metrics and the Schwarz Lemma of the third author show the following:

THEOREM 5.2. Let $X$ be a holomorphic homogeneous regular manifold. Then the Kobayashi metric, the Bergman metric and the Carathéodory metric on $X$ are equivalent.

REMARK 5.2. It follows from the Bers embedding theorem that the Teichmüller space of genus $g$ Riemann surfaces is a holomorphic homogeneous regular manifold if we choose $r=2$ and $R=6$ in Definition 5.1. 


\section{Goodness of canonical metrics}

In his work [19], Mumford defined the goodness condition to study the currents of Chern forms defined by a singular Hermitian metric on a holomorphic bundle over a quasi-projective manifold where he generalized the Hirzebruch's proportionality theorem to noncompact case.

The goodness condition is a growth condition of the Hermitian metric near the compactification divisor of the base manifold. The major property of a good metric is that the currents of its Chern forms define the Chern classes of the bundle. Namely the Chern-Weil theory works in this noncompact case.

Beyond the case of homogeneous bundles over symmetric spaces discussed by Mumford in [19], several natural bundles over moduli spaces of Riemann surfaces give beautiful and useful examples. In [28], Wolpert showed that the metric induced by the hyperbolic metric on the relative dualizing sheaf over the universal curve of moduli space of hyperbolic Riemann surfaces is good. Later it was shown by Trapani [25] that the metric induced by the WP metric on the determinant line bundle of the logarithmic cotangent bundle of the Deligne-Mumford moduli space is good. In both cases, the bundles involved are line bundles. It is easier to estimate the connection and curvature in these cases. Other than these, very few examples of natural good metrics are known.

The goodness of the WP metric has been a long standing open problem. In this section we describe our work in $[\mathbf{1 3}]$ which gives a positive answer to this problem.

We first recall the definition of good metrics and their basic properties described in [19]. Let $\bar{X}$ be a projective manifold of complex dimension $\operatorname{dim}_{\mathbb{C}} \bar{X}=n$. Let $D \subset \bar{X}$ be a divisor of normal crossing and let $X=\bar{X} \backslash D$ be a Zariski open manifold. We let $\Delta_{r}$ be the open disk in $\mathbb{C}$ with radius $r$, let $\Delta=\Delta_{1}, \Delta_{r}^{*}=\Delta_{r} \backslash\{0\}$ and $\Delta^{*}=\Delta \backslash\{0\}$. For each point $p \in D$ we can find a coordinate chart $\left(U, z_{1}, \ldots, z_{n}\right)$ around $p$ in $\bar{X}$ such that $U \cong \Delta^{n}$ and $V=U \cap X \cong\left(\Delta^{*}\right)^{m} \times \Delta^{n-m}$. We assume that $U \cap D$ is defined by the equation $z_{1} \ldots z_{k}=0$. We let $U(r) \cong \Delta_{r}^{n}$ for $0<r<1$ and let $V(r)=U(r) \cap X$.

On the chart $V$ of $X$ we can define a local Poincaré metric:

$$
\omega_{l o c}=\frac{\sqrt{-1}}{2} \sum_{i=1}^{k} \frac{d z_{i} \wedge d \bar{z}_{i}}{2\left|z_{i}\right|^{2}\left(\log \left|z_{i}\right|\right)^{2}}+\frac{\sqrt{-1}}{2} \sum_{i=k+1}^{n} d z_{i} \wedge d \bar{z}_{i}
$$

Now we cover $D \subset \bar{X}$ by such coordinate charts $U_{1}, \ldots, U_{q}$ and let $V_{i}=U_{i} \cap$ $X$. We choose coordinates $z_{1}^{i}, \ldots, z_{n}^{i}$ such that $D \cap U_{i}$ is given by $z_{1}^{i} \ldots z_{m_{i}}^{i}=0$.

A Kähler metric $\omega_{g}$ on $X$ has Poincaré growth if for each $1 \leq i \leq q$ there are constants $0 \leq r_{i} \leq 1$ and $0 \leq c_{i}<C_{i}$ such that $\left.\omega_{g}\right|_{V_{i}\left(r_{i}\right)}$ is equivalent to the local Poincaré metric $\omega_{l o c}^{i}$ :

$$
c_{i} \omega_{l o c}^{i} \leq\left.\omega_{g}\right|_{V_{i}\left(r_{i}\right)} \leq C_{i} \omega_{l o c}^{i} .
$$


In [19] Mumford defined differential forms with Poincaré growth:

Definition 6.1. Let $\eta \in A^{p}(X)$ be a smooth p-form. Then $\eta$ has Poincaré growth if for each $1 \leq i \leq q$ there exists a constant $c_{i}>0$ such that for each point $s \in V_{i}\left(\frac{1}{2}\right)$ and tangent vectors $t_{1}, \ldots, t_{p} \in T_{s} X$ one has

$$
\left|\eta\left(t_{1}, \ldots, t_{p}\right)\right|^{2} \leq c_{i} \prod_{j=1}^{p} \omega_{l o c}^{i}\left(t_{j}, t_{j}\right) .
$$

The $p$-form $\eta$ is good if and only if both $\eta$ and $d \eta$ have Poincaré growth.

REMARK 6.1. It is easy to see that the above definition does not depend on the choice of the cover $\left(U_{1}, \ldots, U_{q}\right)$ but it does depend on the compactification $\bar{X}$ of $X$.

The above definition is local. We now give a global formulation.

Lemma 6.1. Let $\omega_{g}$ be a Kähler metric on $X$ with Poincaré growth. Then a p-form $\eta \in A^{p}(X)$ has Poincaré growth if and only if $\|\eta\|_{g}<\infty$ where $\|\eta\|_{g}$ is the $C^{0}$ norm of $\eta$ with respect to the metric $g$. Furthermore, the fact that $\eta$ has Poincaré growth is independent of the choice of $g$. It follows that if $\eta_{1} \in A^{p}(X)$ and $\eta_{2} \in A^{q}(X)$ have Poincaré growth, then $\eta_{1} \wedge \eta_{2}$ also has Poincaré growth.

Now we collect the basic properties of forms with Poincaré growth as described in $[\mathbf{1 9}]$.

Lemma 6.2. Let $\eta \in A^{p}(X)$ be a form with Poincaré growth. Then $\eta$ defines a p-current on $\bar{X}$. Furthermore, if $\eta$ is good then $d[\eta]=[d \eta]$.

Now we consider a holomorphic vector bundle $\bar{E}$ of rank $r$ over $\bar{X}$. Let $E=\left.\bar{E}\right|_{X}$ and let $h$ be a Hermitian metric on $E$. According to [19] we have

Definition 6.2. The Hermitian metric $h$ is good if for any point $x \in D$, assume $x \in U_{i}$ for some $i$, and any basis $e_{1}, \ldots, e_{r}$ of $\left.\bar{E}\right|_{U_{i}\left(\frac{1}{2}\right)}$, if we let $h_{\alpha \bar{\beta}}=h\left(e_{\alpha}, e_{\beta}\right)$ then there exist positive constants $c_{i}, d_{i}$ such that

(1) $\left|h_{\alpha \bar{\beta}}\right|,(\operatorname{det} h)^{-1} \leq c_{i}\left(\sum_{j=1}^{m_{i}} \log \left|z_{j}\right|\right)^{2 d_{i}} ;$

(2) the 1 -forms $\left(\partial h \cdot h^{-1}\right)_{\alpha \beta}$ are good on $V_{i}\left(\frac{1}{2}\right)$.

REMARK 6.2. A simple computation shows that the goodness of $h$ is independent of the choice of the cover of D. Furthermore, to check whether a metric $h$ is good or not by using the above definition, we only need to check the above two conditions for one choice of the basis $e_{1}, \ldots, e_{r}$.

The most important features of a good metric are 
TheOrem 6.1. Let $h$ be a Hermitian metric on E. Then there is at most one extension of $E$ to $\bar{X}$ for which $h$ is good. Furthermore, if $h$ is a good metric on $E$, then the Chern forms $c_{k}(E, h)$ are good and the current $\left[c_{k}(E, h)\right]=c_{k}(\bar{E}) \in H^{2 k}(\bar{X})$.

See [19] for details. This theorem allows us to compute the Chern classes by using Chern forms of a singular good metric.

Now we look at a special choice of the bundle $E$. In the following we let $\bar{E}=T_{\bar{X}}(-\log D)$ to be the logarithmic tangent bundle and let $E=\left.\bar{E}\right|_{X}$. Let $U$ be one of the charts $U_{i}$ described above and assume $D \cap U$ is given by $z_{1} \ldots z_{m}=0$. Let $V=V_{i}=U_{i} \cap X$. In this case a local frame of $\bar{E}$ restricting to $V$ is given by

$$
e_{1}=z_{1} \frac{\partial}{\partial z_{1}}, \cdots, e_{m}=z_{m} \frac{\partial}{\partial z_{m}}, e_{m+1}=\frac{\partial}{\partial z_{m+1}}, \cdots, e_{n}=\frac{\partial}{\partial z_{n}} .
$$

Let $g$ be any Kähler metric on $X$. It induces a Hermitian metric $\widetilde{g}$ on $E$. In local coordinate $z=\left(z_{1}, \ldots, z_{n}\right)$ we have

$$
\widetilde{g}_{i \bar{j}}= \begin{cases}z_{i} \bar{z}_{j} g_{i \bar{j}} & \text { if } \quad i, j \leq m \\ z_{i} g_{i \bar{j}} & \text { if } \quad i \leq m<j \\ \bar{z}_{j} g_{i \bar{j}} & \text { if } \quad j \leq m<i \\ g_{i \bar{j}} & \text { if } \quad i, j>m .\end{cases}
$$

In the following we denote by $\partial_{i}$ the partial derivative $\frac{\partial}{\partial z_{i}}$. Let

$$
\Gamma_{i k}^{p}=g^{p \bar{q}} \partial_{i} g_{k \bar{q}}
$$

be the Christoffel symbol of the Kähler metric $g$ and let

$$
R_{i k \bar{l}}^{p}=g^{p \bar{j}} R_{i \bar{j} k \bar{l}}=g^{p \bar{j}}\left(-\partial_{k} \partial_{\bar{l}} g_{i \bar{j}}+g^{s \bar{s}} \partial_{k} g_{i \bar{t}} \partial_{\bar{l}} g_{s \bar{j}}\right)
$$

be the curvature of $g$. We define

$$
D_{i}^{k}=\left\{\begin{array}{lll}
\frac{z_{i}}{z_{k}} & \text { if } & i, k \leq m \\
\frac{1}{z_{k}} & \text { if } & k \leq m<i \\
z_{i} & \text { if } & i \leq m<k \\
1 & \text { if } & i, k>m
\end{array}\right.
$$

and we let

$$
\Lambda_{i}=\left\{\begin{array}{lll}
\frac{-1}{\left|z_{i}\right| \log \left|z_{i}\right|} & \text { if } & i \leq m \\
1 & \text { if } & i>m
\end{array} .\right.
$$

Now we give an equivalent local condition of the metric $\widetilde{g}$ on $E$ induced by the Kähler metric $g$ to be good. We have 
Proposition 6.1. The metric $\widetilde{g}$ on $E$ induced by $g$ is good on $V\left(\frac{1}{2}\right)$ if and only if

$$
\begin{aligned}
& \left|\widetilde{g}_{i \bar{j}}\right|,\left|z_{1} \ldots z_{m}\right|^{-2} \operatorname{deg}(g) \leq c\left(\sum_{i=1}^{m} \log \left|z_{i}\right|\right)^{2 d} \text { for some constants } c, d>0 \\
& \left|D_{i}^{k} \Gamma_{i p}^{k}\right|=O\left(\Lambda_{p}\right) \quad \text { for all } 1 \leq i, k, p \leq n \text { except } i=k=p \\
& \left|\frac{1}{t_{i}}+\Gamma_{i i}^{i}\right|=O\left(\Lambda_{i}\right) \quad \text { if } i \leq m \\
& \left|D_{i}^{k} R_{i p \bar{q}}^{k}\right|=O\left(\Lambda_{p} \Lambda_{q}\right) .
\end{aligned}
$$

In $[\mathbf{1 3}]$ we showed the goodness of the WP, Ricci and perturbed Ricci metrics.

TheOREM 6.2. Let $\mathcal{M}_{g}$ be the moduli space of genus $g$ Riemann surfaces. We assume $g \geq 2$. Let $\overline{\mathcal{M}}_{g}$ be the Deligne-Mumford compactification of $\mathcal{M}_{g}$ and let $D=\overline{\mathcal{M}}_{g} \backslash \mathcal{M}_{g}$ be the compactification divisor which is a normal crossing divisor. Let $\bar{E}=T_{\overline{\mathcal{M}}_{g}}(-\log D)$ and let $E=\left.\bar{E}\right|_{\mathcal{M}_{g}}$. Let $\hat{h}, \hat{\tau}$ and $\hat{\widetilde{\tau}}$ be the metrics on $E$ induced by the WP, Ricci and perturbed Ricci metrics respectively. Then $\hat{h}, \hat{\tau}$ and $\hat{\widetilde{\tau}}$ are good in the sense of Mumford.

This theorem is based on very accurate estimates of the connection and curvature forms of these metrics. One of the difficulties is to get accurate estimate of the derivatives of the fiberwise Kähler-Einstein metric in the base direction. We use a refined version of the compound graft metric construction of Wolpert together with the maximum principle to get the desired estimates.

\section{Negativity and naturalness}

In Section 2 we have seen various negative properties of the WP metric. In fact, we showed in $[\mathbf{1 3}]$ that the WP metric is dual Nakano negative. This means the complex curvature operator of the dual metric of the WP metric is positive. We first recall the precise definition of dual Nakano negativity of a Hermitian metric.

Let $(E, h)$ be a Hermitian holomorphic vector bundle of rank $m$ over a complex manifold $M$ of dimension $n$. Let $e_{1}, \ldots, e_{m}$ be a local holomorphic frame of $E$ and let $z_{1}, \ldots, z_{n}$ be local holomorphic coordinates on $M$. The Hermitian metric $h$ has expression $h_{i \bar{j}}=h\left(e_{i}, e_{j}\right)$ locally.

The curvature of $E$ is given by

$$
P_{i \bar{j} \alpha \bar{\beta}}=-\partial_{\alpha} \partial_{\bar{\beta}} h_{i \bar{j}}+h^{p \bar{q}} \partial_{\alpha} h_{i \bar{q}} \partial_{\bar{\beta}} h_{p \bar{j}} .
$$

Definition 7.1. The Hermitian vector bundle $(E, h)$ is Nakano positive if the curvature $P$ defines a Hermitian metric on the bundle $E \otimes$ 
$T_{M}^{1,0}$. Namely, $P_{i \bar{j} \alpha \bar{\beta}} C^{i \alpha} \overline{C^{j \beta}}>0$ for all $m \times n$ nonzero matrices $C$. The bundle $(E, h)$ is Nakano semi-positive if $P_{i \bar{j} \alpha \bar{\beta}} C^{i \alpha} \overline{C^{j \beta}} \geq 0$. The bundle is dual Nakano (semi-)negative if the dual bundle with dual metric $\left(E^{*}, h^{*}\right)$ is Nakano (semi-)positive.

We have proved the following theorem in $[\mathbf{1 3}]$

THEOREM 7.1. Let $\mathcal{M}_{g}$ be the moduli space of Riemann surfaces of genus $g$ where $g \geq 2$. Let $h$ be the WP metric on $\mathcal{M}_{g}$. Then the holomorphic tangent bundle $T^{1,0} \mathcal{M}_{g}$ equipped with the WP metric $h$ is dual Nakano negative.

The dual Nakano negativity is the strongest negativity property of the WP metric. For readers' convenience we include a proof here. The idea is similar to the proof of Proposition 2.2 by using the Green's function of the operator $\square+1$.

ProOF. By definition 7.1 we only need to show that the holomorphic cotangent bundle of the moduli space equipped with the dual metric of the WP metric $\left(T^{*} \mathcal{M}_{g}, h^{*}\right)$ is Nakano positive.

Let $s_{1}, \ldots, s_{3 g-3}$ be any holomorphic coordinates on $\mathcal{M}_{g}$. The dual metric $h^{*}$ is given by $h_{i \bar{j}}^{*}=h^{i \bar{j}}$ locally. If we let $R_{i \bar{j} k \bar{l}}$ be the curvature of the WP metric on $\mathcal{M}_{g}$ and let $P_{i \bar{j} k \bar{l}}$ be the curvature of the metric $h^{*}$, a simple computation shows that

$$
P_{m \bar{n} k \bar{l}}=-h^{i \bar{n}} h^{m \bar{j}} R_{i \bar{j} k \bar{l}} .
$$

Thus if we let $a_{k j}=\sum_{m} h^{m \bar{j}} C^{m k}$, we have

$$
\begin{gathered}
P_{m \bar{n} k \bar{l}} C^{m k} \overline{C^{n l}}=-\sum_{i, j, k, l} R_{i \bar{j} k \bar{l}} a_{k j} \overline{a_{l i}} \\
=-\sum_{i, j, k, l} R_{k \bar{j} i \bar{l}} a_{k j} \overline{a_{l i}}=-\sum_{i, j, k, l} R_{i \bar{j} k \bar{l}} a_{i j} \overline{a_{l k}} .
\end{gathered}
$$

We note that since the matrix $\left[h^{i \bar{j}}\right]$ is invertible, the matrix $\left[a_{i j}\right]$ is nonzero.

Now we fix any point $p \in \mathcal{M}_{g}$ and let $X=X_{p}$ be the corresponding Riemann surface. Let $s_{1}, \ldots, s_{3 g-3}$ be any holomorphic coordinates around $p$ and let $A_{i}$ be the harmonic Beltrami differential corresponding to $\frac{\partial}{\partial s_{i}}$. By using the curvature formula (2.2) of the WP metric, we only need to show that

$$
\sum_{i, j, k, l} \int_{X}\left(e_{i \bar{j}} f_{k \bar{l}}+e_{i \bar{l}} f_{k \bar{j}}\right) a_{i j} \overline{a_{l k}} d v>0
$$

for any nonzero matrix $\left[a_{i j}\right]$. 
We let $T=(\square+1)^{-1}$ be the Green operator and we recall that

$$
e_{i \bar{j}}=T\left(f_{i \bar{j}}\right)
$$

where $f_{i \bar{j}}=A_{i} \overline{A_{j}}$. Let $B_{j}=\sum_{i=1}^{n} a_{i j} A_{i}$. Then the inequality (7.1) is equivalent to

$$
\sum_{j, k} \int_{X}\left(T\left(B_{j} \overline{A_{j}}\right) A_{k} \overline{B_{k}}+T\left(B_{j} \overline{B_{k}}\right) A_{k} \overline{A_{j}}\right) d v>0
$$

To prove the inequality (7.2), we let $\mu=\sum_{j} B_{j} \overline{A_{j}}=\sum_{i, j} a_{i j} f_{i \bar{j}}$. Then for the first term of formula (7.2) we have

$$
\sum_{j, k} \int_{X} T\left(B_{j} \overline{A_{j}}\right) A_{k} \overline{B_{k}} d v=\int_{X} T(\mu) \bar{\mu} d v \geq 0
$$

where equality holds if and only if $\mu=0$.

To check the second term, we let $G(z, w)$ be the Green's function of the operator T. Similar to the proof of Proposition 2.2 we know that the Green's function $G \geq 0$. Furthermore,

$$
m(\{(z, w) \in X \times X \mid G(z, w)=0\})=0
$$

where $m$ is the Lebesgue measure on $X \times X$.

We consider the smooth line bundle $L=T_{X}^{1,0} \otimes \Omega_{X}^{0,1}$ over $X$. Let $\pi_{1}, \pi_{2}$ : $X \times X \rightarrow X$ be the projection maps to the first and second factors respectively. We let

$$
H(z, w)=\sum_{j} \overline{A_{j}}(z) B_{j}(w)
$$

Then $H(z, w)$ can be viewed as a section of the line bundle $\pi_{1}^{*}(\bar{L}) \otimes \pi_{2}^{*}(L)$ over $X \times X$. It has a natural $L^{2}$ norm. It follows that the second term of (7.2) is

$$
\begin{aligned}
& \sum_{j, k} \int_{X} T\left(B_{j} \overline{B_{k}}\right) A_{k} \overline{A_{j}} d v \\
& \quad=\sum_{j, k} \int_{X} \int_{X} G(z, w) B_{j}(w) \overline{B_{k}}(w) A_{k}(z) \overline{A_{j}}(z) d v(w) d v(z) \\
& \quad=\int_{X} \int_{X} G(z, w)|H(z, w)|^{2} d v(w) d v(z) \geq 0 .
\end{aligned}
$$

Since the Green's function $G(z, w)$ is positive away from a set of measure zero, the equality in the above inequality holds if and only if $H(z, w)=0$. Since $A_{1}, \ldots, A_{3 g-3}$ is a basis of $\mathbb{H}^{0,1}\left(X, T_{X}^{1,0}\right)$ and the matrix $\left[a_{i j}\right]$ is nonzero, we know that $B_{1}, \ldots, B_{3 g-3}$ do not vanish simultaneously. Thus we 
can find a point $w$ such that $B_{1}(w), \ldots, B_{3 g-3}(w)$ is not the zero vector. Now we fix the point $w$. Since $H(z, w)=0$ for all $z$ we know that $\bar{A}_{1}, \ldots, \bar{A}_{3 g-3}$ are linearly dependent which is a contradiction. Thus the equality of formula (7.3) cannot hold. This implies that the left side of formula (7.2) is strictly positive and thus the WP metric is dual Nakano negative.

Now we look at the naturalness of the canonical metrics on the moduli space. We let $\mathcal{M}_{g}$ be the moduli space of genus $g$ curves where $g \geq 2$ and let $\overline{\mathcal{M}}_{g}$ be its Deligne-Mumford compactification. We fix a point $p \in \overline{\mathcal{M}}_{g} \backslash \mathcal{M}_{g}$ of codimension $m$ and let $X=X_{p}$ be the corresponding stable nodal curve. The moduli space $\mathcal{M}(X)$ of the nodal surface $X$ is naturally embedded into $\overline{\mathcal{M}}_{g}$. Furthermore, since each element $Y$ in $\mathcal{M}(X)$ corresponds to a hyperbolic Riemann surface when we remove the nodes from $Y$, the complement can be uniformized by the upper half plane and thus there is a unique complete Kähler-Einstein metric on $Y$ whose Ricci curvature is -1 . We note that the moduli space $\mathcal{M}(X)$ can be viewed as an irreducible component of the intersection of the compactification divisors.

By the discussion in Section 2 there is a natural WP metric $\hat{h}$ on $\mathcal{M}(X)$. The curvature formula (2.2) is still valid for this WP metric and it is easy to see that the Ricci curvature of the WP metric $\hat{h}$ is negative. We can take $\hat{\tau}=-\operatorname{Ric}\left(\omega_{\hat{h}}\right)$ to be the Kähler form of a Kähler metric on $\mathcal{M}(X)$. This is the Ricci metric $\hat{\tau}$ on $\mathcal{M}(X)$.

In [17] Masur showed that the WP metric $h$ on $\mathcal{M}_{g}$ extends to $\overline{\mathcal{M}}_{g}$ and its restriction to $\mathcal{M}(X)$ via the natural embedding $\mathcal{M}(X) \hookrightarrow \overline{\mathcal{M}}_{g}$ coincides with the WP metric $\hat{h}$ on $\mathcal{M}(X)$. This implies the WP metric is natural. In [31] Wolpert showed that the WP Levi-Civita connection restricted to directions which are almost tangential to the compactification divisors limits to the lower dimensional WP Levi-Civita connection. In [13] we proved the naturalness of the Ricci metric.

Theorem 7.2. The Ricci metric on $\mathcal{M}_{g}$ extends to $\overline{\mathcal{M}}_{g}$ in nondegenerating directions. Furthermore, the restriction of the extension of $\tau$ to $\mathcal{M}(X)$ coincides with $\hat{\tau}$, the Ricci metric on $\mathcal{M}(X)$.

\section{The Kähler-Ricci flow and Kähler-Einstein metric on the moduli space}

The existence of the Kähler-Einstein metric on the Teichmüller space was based on the work of Cheng-Yau since the Teichmüller space is pseudoconvex. By the uniqueness we know that the Kähler-Einstein metric is invariant under the action of the mapping class group and thus is also the KählerEinstein metric on the moduli space. It follows from the later work of Yau that the Kähler-Einstein metric is complete. However, the detailed properties of the Kähler-Einstein metric remain unknown. 
In $[\mathbf{1 0}]$ we proved the strongly bounded geometry property of the KählerEinstein metric. We showed

THEOREM 8.1. The Kähler-Einstein metric on the Teichmüller space $\mathcal{T}_{g}$ has strongly bounded geometry. Namely, the curvature and its covariant derivatives of the Kähler-Einstein metric are bounded and the injectivity radius of the Kähler-Einstein metric is bounded from below.

This theorem was proved in two steps. Firstly, we deform the Ricci metric via the Kähler-Ricci flow

$$
\left\{\begin{array}{l}
\frac{\partial g_{i \bar{j}}}{\partial t}=-\left(R_{i \bar{j}}+g_{i \bar{j}}\right) \\
g_{i \bar{j}}(0)=\tau_{i \bar{j}}
\end{array}\right.
$$

Let $h=g(s)$ be the deformed metric at time $s \ll 1$. By the work of Shi [23] we know that the metric $h$ is equivalent to the initial metric $\tau$ and is cohomologous to $\tau$ in the sense of currents. Thus $h$ is complete and has Poincaré growth. Furthermore, the curvature and covariant derivatives of $h$ are bounded.

We then use the metric $h$ as a background metric to derive a priori estimates for the Kähler-Einstein metric by using the Monge-Amperé equation

$$
\frac{\operatorname{det}\left(h_{i \bar{j}}+u_{i \bar{j}}\right)}{\operatorname{det} h_{i \bar{j}}}=e^{u+F}
$$

where $F$ is the Ricci potential of the metric $h$. If we denote by $g$ the KählerEinstein metric and let

$$
S=g^{i \bar{j}} g^{k \bar{l}} g^{p \bar{q}} u_{; i \bar{q} k} u_{; \bar{j} p \bar{l}}
$$

and

$$
V=g^{i \bar{j}} g^{k \bar{l}} g^{p \bar{q}} g^{m \bar{n}} u_{; i \bar{q} k \bar{n}} u_{; \bar{j} p \bar{l} m}+g^{i \bar{j}} g^{k \bar{l}} g^{p \bar{q}} g^{m \bar{n}} u_{; i \bar{n} k p} u_{; \bar{j} m m \bar{l} \bar{q}}
$$

to be the third and fourth order quantities respectively. We have

$$
\begin{aligned}
\Delta^{\prime}[(S+\kappa) V] \geq & C_{1}[(S+\kappa) V]^{2}-C_{2}[(S+\kappa) V]^{\frac{3}{2}}-C_{3}[(S+\kappa) V] \\
& -C_{4}[(S+\kappa) V]^{\frac{1}{2}}
\end{aligned}
$$

where $\Delta^{\prime}$ is the Laplace operator of the Kähler-Einstein metric $g$ and $C_{1}>0$.

It follows from the mean value inequality that $S$ is bounded. Furthermore, by the above estimate and the maximum principle we know $V$ is bounded. In fact this method works for all higher order derivatives of $u$ and we deduce that the Kähler-Einstein metric has strongly bounded geometry.

The Kähler-Ricci flow and the goodness are closely tied together. Firstly, since the most important feature of a Mumford good metric is that the Chern-Weil theory still holds, we say metrics with this property are intrinsic good. In $[\mathbf{1 4}]$ we showed 
TheOREM 8.2. Let $\bar{X}$ be a projective manifold with $\operatorname{dim}_{\mathbb{C}} \bar{X}=n$. Let $D \subset \bar{X}$ be a divisor with normal crossings, let $X=\bar{X} \backslash D$, let $\bar{E}=T_{\bar{X}}(-\log D)$ and let $E=\left.\bar{E}\right|_{X}$.

Let $\omega_{g}$ be a Kähler metric on $X$ with bounded curvature and Poincaré growth. Assume Ric $\left(\omega_{g}\right)+\omega_{g}=\partial \bar{\partial} f$ where $f$ is a bounded smooth function. Then

- There exists a unique Kähler-Einstein metric $\omega_{K E}$ on $X$ and the Kähler-Einstein metric has Poincaré growth.

- The curvature and covariant derivatives of curvature of the KählerEinstein metric are bounded.

- If $\omega_{g}$ is intrinsic good, then $\omega_{K E}$ is intrinsic good. Furthermore, all metrics along the paths of continuity and Kähler-Ricci flow are intrinsic good.

\section{Applications}

In this section we briefly look at some geometric applications of the canonical metrics. The first application of the control of the Kähler-Einstein metric is the stability of the logarithmic cotangent bundle of the DeligneMumford moduli space. In [10] we proved

TheOREM 9.1. Let $\bar{E}=T_{\mathcal{M}_{g}}^{*}(\log D)$ be the logarithmic cotangent bundle. Then $c_{1}(\bar{E})$ is positive and $\bar{E}$ is slope stable with respect to the polarization $c_{1}(\bar{E})$.

An immediate consequence of the intrinsic goodness of the Kähler-Einstein metric is the Chern number inequality. We have

TheOrEm 9.2. Let $\bar{E}=T_{\overline{\mathcal{M}}_{g}}(-\log D)$ be the logarithmic tangent bundle of the moduli space. Then

$$
c_{1}(\bar{E})^{2} \leq \frac{6 g-4}{3 g-3} c_{2}(\bar{E})
$$

It follows directly from the dual Nakano negativity and the Mumford goodness of the WP metric that the Chern numbers of the local cotangent bundle are positive. We have

THEOREM 9.3. The Chern numbers of the logarithmic cotangent bundle $T_{\mathcal{M}_{g}}^{*}(\log D)$ of the moduli spaces of Riemann surfaces are all positive.

The dual Nakano negativity of a Hermitian metric on a bundle over a compact manifold gives strong vanishing theorems by using Bochner techniques. However, in our case the base variety $\mathcal{M}_{g}$ is only quasi-projective. Thus we can only describe vanishing theorems of the $L^{2}$ cohomology. 
In [21], Saper showed that the $L^{2}$ cohomology of the moduli space equipped with the Weil-Petersson metric can be identified with the ordinary cohomology of the Deligne-Mumford moduli space. Our situation is more subtle since the natural object to be considered in our case is the tangent bundle valued $L^{2}$ cohomology. Parallel to Saper's work, we proved in $[\mathbf{1 4}]$

THEOREM 9.4. We have the following natural isomorphism

$$
H_{(2)}^{*}\left(\left(\mathcal{M}_{g}, \omega_{\tau}\right),\left(T_{\mathcal{M}_{g}}, \omega_{W P}\right)\right) \cong H^{*}\left(\overline{\mathcal{M}}_{g}, T_{\overline{\mathcal{M}}_{g}}(-\log D)\right) .
$$

Now we combine the above result with the dual Nakano negativity of the WP metric. In [14] we proved the following Nakano-type vanishing theorem

THEOREM 9.5. The $L^{2}$ cohomology groups vanish:

$$
H_{(2)}^{0, q}\left(\left(\mathcal{M}_{g}, \omega_{\tau}\right),\left(T_{\mathcal{M}_{g}}, \omega_{W P}\right)\right)=0
$$

unless $q=3 g-3$.

As a direct corollary we have

COROLlary 9.1. The pair $\left(\overline{\mathcal{M}}_{g}, D\right)$ is infinitesimally rigid.

Another important application of the properties of the Ricci, perturbed Ricci and Kähler-Einstein metrics is the Gauss-Bonnet theorem on the noncompact moduli space. Together with L. Ji, in [6] we showed

TheOrem 9.6. The Gauss-Bonnet theorem holds on the moduli space equipped with the Ricci, perturbed Ricci or Kähler-Einstein metrics:

$$
\int_{\mathcal{M}_{g}} c_{n}\left(\omega_{\tau}\right)=\int_{\mathcal{M}_{g}} c_{n}\left(\omega_{\widetilde{\tau}}\right)=\int_{\mathcal{M}_{g}} c_{n}\left(\omega_{K E}\right)=\chi\left(\mathcal{M}_{g}\right)=\frac{B_{2 g}}{4 g(g-1)} .
$$

Here $\chi\left(\mathcal{M}_{g}\right)$ is the orbifold Euler characteristic of $\mathcal{M}_{g}$ and $n=3 g-3$.

The explicit topological computation of the Euler characteristic of the moduli space is due to Harer-Zagier [5]. See also the work of Penner [20].

As an application of the Mumford goodness of the WP metric and the Ricci metric we have

THEOREM 9.7 .

$$
\chi\left(T_{\overline{\mathcal{M}}_{g}}(-\log D)\right)=\int_{\mathcal{M}_{g}} c_{n}\left(\omega_{\tau}\right)=\int_{\mathcal{M}_{g}} c_{n}\left(\omega_{W P}\right)=\frac{B_{2 g}}{4 g(g-1)}
$$

where $n=3 g-3$. 
It is very hard to prove the Gauss-Bonnet theorem for the WP metric directly since the WP metric is incomplete and its curvature is not bounded. The proof is based substantially on the Mumford goodness of the WP metric.

By using the goodness of canonical metrics this theorem also gives an explicit expression of the top log Chern number of the moduli space.

THEOREM 9.8 .

$$
\chi\left(\overline{\mathcal{M}}_{g}, T_{\overline{\mathcal{M}}_{g}}(-\log D)\right)=\chi\left(\mathcal{M}_{g}\right)=\frac{B_{2 g}}{4 g(g-1)} .
$$

\section{Deformation of pluricanonical forms}

In this section we discuss an iteration method to deform holomorphic sections of the pluricanonical bundle of a hyperbolic Riemann surface.

We let $\mathcal{M}=\mathcal{M}_{g}$ be the moduli space of genus $g$ curves and let

$$
\pi: \mathfrak{X} \rightarrow \mathcal{M}
$$

be the universal family. Here we assume $g \geq 2$. For each point $p \in \mathcal{M}_{g}$ we let $X_{p}=\pi^{-1}(p)$ be the fiber. We recall Remark 2.1 that we always work on the local manifold cover of the moduli space when we look at local deformation theory.

Let $\omega_{\mathfrak{X} / \mathcal{M}}$ be the relative dualizing sheaf. For any $m \geq 1$ we consider the holomorphic vector bundle

$$
E=E_{m}=R^{0} \omega_{\mathfrak{X} / \mathcal{M}}^{m}
$$

over $\mathcal{M}_{g}$.

When $m=1$ the bundle $E_{1}$ is just the Hodge bundle. The method in the section will give a simple formula of the curvature of the $L^{2}$ metric on the Hodge bundle. When $m=2$ we know that $E_{2} \cong \Omega^{1,0} \mathcal{M}_{g}$ is the holomorphic cotangent bundle of $\mathcal{M}_{g}$.

For each point $q \in \mathcal{M}$, if $m \geq 2$, by Serre duality we know that

$$
h^{1}\left(X_{p}, K_{X_{p}}^{m}\right)=h^{0}\left(X_{p}, K_{X_{p}}^{1-m}\right)=0
$$

since $1-m<0$ and $K_{X_{p}}$ is a positive line bundle. It follows from the Riemann-Roch theorem that

$$
\operatorname{rank}\left(E_{m}\right)=h^{0}\left(X_{p}, K_{X_{p}}^{m}\right)=\int_{X_{p}} \operatorname{ch}\left(K_{X_{p}}^{m}\right) \operatorname{Td}\left(X_{p}\right)=(2 m-1)(g-1)
$$

in the case $m \geq 2$. When $m=1$ we know that $\operatorname{rank}\left(E_{1}\right)=g$.

10.1. Kuranishi family. We first look at the local Kuranishi family of Riemann surfaces with a particular choice of gauge. We fix a point $0 \in \mathcal{M}_{g}$ and let $X_{0}$ be a Riemann surface representing this point. Let $\Sigma$ be the 
underlying smooth surface. In this case we can write $X_{0}=\left(\Sigma, J_{0}\right)$. Let $\lambda$ be the Kähler-Einstein metric on $X_{0}$. We always use the Kähler-Einstein metric on Riemann surfaces in this section.

Let $\varphi \in \mathbb{H}^{0,1}\left(X_{0}, T_{X_{0}}^{1,0}\right)$ be a harmonic Beltrami differential such that

$$
\sup _{X_{0}}|\varphi|<1
$$

Let $z$ be any holomorphic local coordinate on $X_{0}$. In this coordinates we have $\varphi=\varphi(z) d \bar{z} \otimes \frac{\partial}{\partial z}$. Let $X_{\varphi}=\left(\Sigma, J_{\varphi}\right)$ be the Riemann surface such that for each point $q \in \Sigma$ we have

$$
\Omega_{q}^{1,0} X_{\varphi}=\mathbb{C}\{d z+\varphi d \bar{z}\}
$$

and

$$
\Omega_{q}^{0,1} X_{\varphi}=\mathbb{C}\{d \bar{z}+\bar{\varphi} d z\}
$$

where $\Omega_{q}^{1,0} X_{0}=\mathbb{C}\{d z\}$ and $\Omega_{q}^{0,1} X_{0}=\mathbb{C}\{d \bar{z}\}$.

In this case a local smooth function $w$ is holomorphic with respect to the complex structure $J_{\varphi}$ if and only if

$$
\frac{\partial w}{\partial \bar{z}}=\varphi(z) \frac{\partial w}{\partial z}
$$

Now we let $n=3 g-3$ and we fix a basis $\varphi_{1}, \ldots, \varphi_{n} \in \mathbb{H}^{0,1}\left(X_{0}, T_{X_{0}}^{1,0}\right)$ of harmonic Beltrami differentials on $X_{0}$. Let $\Delta \subset \mathbb{C}^{n}$ be the ball of radius $\varepsilon$. We consider the smooth product manifold $\mathcal{U}=\Sigma \times \Delta$ and let $\pi: \mathcal{U} \rightarrow \Delta$ be the natural projection map.

We let $t=\left(t_{1}, \ldots, t_{n}\right)$ be the natural coordinates on $\Delta$ and let

$$
\varphi(t)=\sum_{i=1}^{n} t_{i} \varphi_{i} .
$$

We choose $\varepsilon>0$ small enough such that

$$
\sup _{X_{0}}|\varphi(t)|<1
$$

for each $t \in \Delta$. We now put a complex structure on each fiber

$$
\pi^{-1}(t) \cong \Sigma
$$

As described above, for each $t \in \Delta$ we let $J_{t}=J_{\varphi(t)}$ and denote by

$$
X_{t}=\left(\Sigma, J_{t}\right)
$$

the corresponding Riemann surface. The family $\pi: \mathcal{U} \rightarrow \Delta$ is a Kuranishi family of the central fiber $X_{0}$. We refer to such a family $\pi: \mathcal{U} \rightarrow \Delta$ the canonical Kuranishi family of $X_{0}$. 
REMARK 10.1. In fact the canonical Kuranishi family was constructed by imposing the Kuranishi gauge. In [8] Kuranishi considered the following equations on a Kähler manifold $X$

$$
\left\{\begin{array}{l}
\bar{\partial} \varphi=\frac{1}{2}[\varphi, \varphi] \\
\bar{\partial}^{*} \varphi=0 .
\end{array}\right.
$$

Here the first equation is the integrability condition and the second equation is the Kuranishi gauge. In our case the first equation is automatically satisfied since the dimension of $X$ is 1 . The Kuranishi gauge is equivalent to the fact that $\varphi$ is harmonic. Of course we use the Kähler-Einstein metric on $X$.

10.2. Deformation of holomorphic sections of pluricanonical bundle. We now describe an iteration procedure of deform holomorphic sections of the relative pluricanonical bundle. Namely we will construct local holomorphic sections of the bundle $E_{m}$.

We first fix a Riemann surface $X_{0}$ representing a point $0 \in \mathcal{M}_{g}$ and we let $\pi: \mathcal{U} \rightarrow \Delta$ be a canonical Kuranishi family of $X_{0}$. For each $t \in \Delta$ we let $\varphi=\varphi(t)$ as described above. The Beltrami differential $\varphi$ induces a map

$$
\sigma=\sigma_{t}: A^{0}\left(X_{0}, K_{X_{0}}^{m}\right) \rightarrow A^{0}\left(X_{t}, K_{X_{t}}^{m}\right)
$$

in the following way. If $\psi \in A^{0}\left(X_{0}, K_{X_{0}}^{m}\right)$ with local expression

$$
\psi=f(z) d z^{m}
$$

then we define

$$
\sigma(\psi)=f(z)(d z+\varphi d \bar{z})^{m}
$$

Lemma 10.1. The map $\sigma$ is well-defined and is a bijective linear map.

Proof. To see that $\sigma$ is well-defined we only need to check that the definition of $\sigma$ is independent of the choice of local coordinate. This follows directly from formulas (10.2) and (10.3). It is also clear that $\sigma$ is linear and is injective.

To show that $\sigma$ is surjective, we pick an element $\psi_{0} \in A^{0}\left(X_{0}, K_{X_{0}}^{m}\right)$ such that $\psi_{0}$ is nowhere vanishing. This can be done by using partition of unity. By the definition of $\sigma$ we know that $\sigma\left(\psi_{0}\right) \in A^{0}\left(X_{t}, K_{X_{t}}^{m}\right)$ is nowhere vanishing. Thus for any element $\tau \in \in A^{0}\left(X_{t}, K_{X_{t}}^{m}\right)$ there exists a unique function $\mu \in C^{\infty}(\Sigma)$ such that $\tau=\mu \sigma\left(\psi_{0}\right)$. If follows that $\sigma\left(\mu \psi_{0}\right)=\tau$ and thus $\sigma$ is surjective.

Now we describe the iteration procedure to construct local holomorphic sections of $E_{m}$. We first consider the case of one-parameter family. We fix a Riemann surface $X_{0}$ and a harmonic Beltrami differential $\varphi \in \mathbb{H}^{0,1}\left(X_{0}, T_{X_{0}}^{1,0}\right)$. 
Consider the family of Riemann surfaces $\left\{X_{t}\right\}$ where $X_{t}=\left(\Sigma, J_{t}\right)$ where $J_{t}=J_{t \varphi}$ for all $|t|<\varepsilon$.

Given a holomorphic section $\psi_{0} \in H^{0}\left(X_{0}, K_{X_{0}}^{m}\right)$ we are looking for a convergent power series

$$
\psi_{t}=\psi_{0}+\sum_{k=1}^{\infty} t^{k} \psi_{k}
$$

where $\psi_{k} \in A^{0}\left(X_{0}, K_{X_{0}}^{m}\right)$ such that

$$
\sigma_{t}\left(\psi_{t}\right) \in H^{0}\left(X_{t}, K_{X_{t}}^{m}\right)
$$

is holomorphic with respect to the complex structure $J_{t}$ for each $t$ with $|t|<\varepsilon$.

We first look at the case $m=1$. We have

Lemma 10.2. Let $\varphi$ be any harmonic Beltrami differential on $X_{0}$ with respect to the Kähler-Einstein metric. We assume $\sup _{X_{0}}|\varphi|<1$. Let $X_{\varphi}$ be the Riemann surface obtained by deforming the complex structure on $X_{0}$ via $\varphi$. Let $\psi \in A^{0}\left(X_{0}, K_{X_{0}}\right)$ be a smooth $(1,0)$-form. Then

$$
\sigma(\psi) \in H^{0}\left(X_{\varphi}, K_{X_{\varphi}}\right)
$$

is holomorphic with respect to the complex structure $J_{\varphi}$ if and only if

$$
\bar{\partial} \psi+\partial(\varphi\lrcorner \psi)=0
$$

where $\partial$ and $\bar{\partial}$ are operators on $X_{0}$.

Proof. For any point $p \in X_{0}$ let $z$ be any holomorphic local coordinate around $p$ with respect to the complex structure on $X_{0}$ and let $w$ be any holomorphic local coordinate around $p$ with respect to the complex structure on $X_{\varphi}$.

Let $a(z)=\frac{\partial w}{\partial z}$. By formula (10.1) we know that $\frac{\partial w}{\partial \bar{z}}=a \varphi$ and

$$
\frac{\partial a}{\partial \bar{z}}=\frac{\partial}{\partial z} \frac{\partial w}{\partial \bar{z}}=\partial_{z} a \varphi+a \partial_{z} \varphi
$$

Since $d w=a(d z+\varphi d \bar{z})$, if we let $\psi=f(z) d z$ locally, then $\sigma(\psi)=\left(f a^{-1}\right) d w$. Thus $\sigma(\psi)$ is holomorphic with respect to the complex structure $J_{\varphi}$ if any only if $f a^{-1}$ is a local holomorphic function with respect to the complex structure $J_{\varphi}$. By formula (10.1) we know that $\sigma(\psi)$ is holomorphic with respect to the complex structure $J_{\varphi}$ if any only if

$$
\partial_{\bar{z}}\left(f a^{-1}\right)=\varphi \partial_{z}\left(f a^{-1}\right) .
$$

By using formula (10.7) we see that equation (10.8) is equivalent to (10.6).

Now we construct the power series (10.4). 
Proposition 10.1. Given any harmonic Beltrami differential $\varphi$ on $X_{0}$ we let $X_{t}=\left(\Sigma, J_{t \varphi}\right)$. Then for any holomorphic 1 -form $\psi_{0}$ on $X_{0}$ there is a unique power series $\psi_{t}=\psi_{0}+\sum_{k=1}^{\infty} t^{k} \psi_{k}$ which converges for $|t|<\varepsilon$ such that

(1) $\sigma_{t}\left(\psi_{t}\right) \in H^{0}\left(X_{t}, K_{X_{t}}\right)$ is holomorphic with respect to the complex structure $J_{t \varphi}$.

(2) For each $k \geq 1$ the $(1,0)$-form $\psi_{k}$ on $X_{0}$ is $\partial$-exact.

Proof. By Lemma 10.2 we know that $\psi_{t}$ must satisfy the equation

$$
\left.\bar{\partial} \psi_{t}+t \partial(\varphi\lrcorner \psi_{t}\right)=0 .
$$

By looking at the coefficients of $t^{k}$ we know that the above equation is equivalent to

$$
\left\{\begin{array}{l}
\bar{\partial} \psi_{0}=0 \\
\left.\bar{\partial} \psi_{k}=-\partial(\varphi\lrcorner \psi_{k-1}\right) \quad \text { for each } k \geq 1
\end{array}\right.
$$

We first find the formal power series $\psi_{t}$. Since $\psi_{0}$ is holomorphic we know that the first equation in (10.10) holds. Now we solve the second equation by induction. For each $k \geq 1$ since $\left.-\partial(\varphi\lrcorner \psi_{k-1}\right)$ is a $(1,1)$-form on $X_{0}$ we know that $\left.\bar{\partial}\left(-\partial(\varphi\lrcorner \psi_{k-1}\right)\right)=0$. Furthermore, since $\left.-\partial(\varphi\lrcorner \psi_{k-1}\right)$ is $\partial$-exact, its projection to the space of harmonic forms is 0 .

By Hodge theory we know that if we let

$$
\left.\psi_{k}=-\bar{\partial}^{*} G \partial(\varphi\lrcorner \psi_{k-1}\right)
$$

where $G$ is the Green operator, then

$$
\begin{aligned}
\bar{\partial} \psi_{k} & \left.\left.=-\overline{\partial \partial}^{*} G \partial(\varphi\lrcorner \psi_{k-1}\right)=-\Delta G \partial(\varphi\lrcorner \psi_{k-1}\right) \\
& \left.\left.=-(I-\mathbb{H}) \partial(\varphi\lrcorner \psi_{k-1}\right)=-\partial(\varphi\lrcorner \psi_{k-1}\right) .
\end{aligned}
$$

Thus $\psi_{k}$ defined by (10.11) is a solution of the second equation of (10.10). Furthermore, by the Kähler identity we know that $\left.\psi_{k}=\partial \bar{\partial}^{*} G(\varphi\lrcorner \psi_{k-1}\right)$ which implies that $\psi_{k}$ is $\partial$-exact.

To prove the convergence we notice that

$$
\| \varphi\lrcorner \psi_{k}\left\|_{C^{r, \alpha}} \leq C\right\| \varphi\left\|_{C^{r, \alpha}}\right\| \psi_{k} \|_{C^{r, \alpha}}
$$

and

$$
\left.\left\|\psi_{k}\right\|_{C^{r, \alpha}} \leq C_{1} \| \varphi\right\lrcorner \psi_{k-1}\left\|_{C^{r, \alpha}} \leq C_{2}\right\| \varphi\left\|_{C^{r, \alpha}}\right\| \psi_{k-1} \|_{C^{r, \alpha}}
$$

where we use formula (10.11). Thus the formal power series (10.4) converges smoothly when $|t|$ is small enough.

A direct corollary of the above proposition is the expansion of the family $\sigma_{t}\left(\psi_{t}\right)$. 
COROLlary 10.1. With the same assumption as in the above proposition the family $\sigma_{t}\left(\psi_{t}\right)$ can be written as a convergent power series of $t$ with coefficients in $A^{1}\left(X_{0}\right)$. Furthermore, we have

$$
\left.\left.\sigma_{t}\left(\psi_{t}\right)=\psi_{0}+t(\varphi\lrcorner \psi_{0}-\bar{\partial}^{*} G \partial(\varphi\lrcorner \psi_{0}\right)\right)+O\left(t^{2}\right)
$$

Proof. This corollary follows from the proof of Proposition 10.2 and the definition of $\sigma_{t}$ directly.

On the Hodge bundle $E_{1}$ there is a natural $L^{2}$ metric. Let $e_{1}, \ldots, e_{g}$ be a local holomorphic frame of $E_{1}$. Then the $L^{2}$ metric is given by

$$
M_{\alpha \bar{\beta}}(p)=\frac{\sqrt{-1}}{2} \int_{X_{p}} e_{\alpha} \wedge \bar{e}_{\beta} .
$$

By using the expansion (10.12) we can easily derive the curvature formula of the $L^{2}$ metric on the Hodge bundle.

Proposition 10.2. For any point $0 \in \mathcal{M}_{g}$ let $X_{0}$ be a Riemann surface representing this point and let $\varphi_{1}, \ldots, \varphi_{n} \in \mathbb{H}^{0,1}\left(X_{0}, T_{X_{0}}^{1,0}\right)$ be a basis of harmonic Beltrami differentials on $X_{0}$. Let $\pi: \mathcal{U} \rightarrow \Delta$ be the corresponding canonical Kuranishi family. Let $\psi^{1}, \ldots, \psi^{g} \in H^{1,0}\left(X_{0}\right)$ be an orthonormal basis with respect to the $L^{2}$ metric. Then the curvature of the $L^{2}$ metric is given by

$$
\left.\left.R_{\alpha \bar{\beta} i \bar{j}}=\frac{\sqrt{-1}}{2} \int_{X_{0}}\left(\overline{\left.\left(\varphi_{j}\right\lrcorner \psi^{\beta}\right)} \wedge\left(\varphi_{i}\right\lrcorner \psi^{\alpha}\right)-\bar{\partial}^{*} G \partial\left(\varphi_{i}\right\lrcorner \psi^{\alpha}\right) \wedge \partial^{*} G \bar{\partial} \overline{\left.\left(\varphi_{j}\right\lrcorner \psi^{\beta}\right)}\right) .
$$

Proof. This proposition follows directly from the general expansion of type (10.12). For any given holomorphic $(1,0)$-form $\psi_{0}$ on $X_{0}$, if we let $t=\left(t_{1}, \ldots, t_{n}\right)$ then we have

$$
\left.\left.\sigma_{t}\left(\psi_{t}\right)=\psi_{0}+\sum_{i=1}^{n} t_{i}\left(\varphi_{i}\right\lrcorner \psi_{0}-\bar{\partial}^{*} G \partial\left(\varphi_{i}\right\lrcorner \psi_{0}\right)\right)+O\left(t^{2}\right) .
$$

This expansion will give us the Taylor expansion of the $L^{2}$ metric $M_{\alpha \bar{\beta}}$. The proposition follows from comparing the coefficients of terms $t_{i} \bar{t}_{j}$ in the Taylor expansion.

REMARK 10.2. We note that the iteration method we described here lead to a complete local expansion of the period map. For geometry of the period domain please see [22] and [4] for details.

Furthermore, we consider the Sternberg lemma on a general n-dimensional manifold $M$. If $\varphi, \tau \in A^{0,1}\left(M, T_{M}^{1,0}\right)$ are two smooth Beltrami 
differentials and $\Omega \in A^{n, 0}(M)$ is a smooth $(n, 0)$-form, then

$$
[\varphi, \tau]\lrcorner \Omega=-\partial(\varphi \wedge \tau\lrcorner \Omega)+\varphi\lrcorner \partial(\tau\lrcorner \Omega)+\tau\lrcorner \partial(\varphi\lrcorner \Omega) .
$$

By using the Sternberg lemma this method can be used to construct canonical sections of the relative canonical bundle of any smooth family of Kähler manifolds of arbitrary dimension.

Now we look at the general case $m \geq 2$. In these cases we need to use the connection of the metric on $K_{X}^{m}$ induced by the Kähler-Einstein metric on the Riemann surface.

We fix a Riemann surface $X_{0}$ and let $\lambda$ be the Kähler-Einstein metric on $X_{0}$. It is clear that $\lambda^{-m}$ is a Hermitian metric on the line bundle $K_{X_{0}}^{m}$. Let $\nabla$ be the corresponding metric connection.

Similar to Lemma 10.2 we first give a necessary and sufficient condition that $\sigma_{t}\left(\psi_{t}\right)$ is holomorphic with respect to the complex structure $J_{t}$.

Lemma 10.3. Let $\varphi$ be any harmonic Beltrami differential on $X_{0}$ with respect to the Kähler-Einstein metric such that $\sup _{X_{0}}|\varphi|<1$. Let $X_{\varphi}$ be the Riemann surface obtained by deforming the complex structure on $X_{0}$ via $\varphi$.

Let $\psi \in A^{0}\left(X_{0}, K_{X_{0}}^{m}\right)$ be a smooth pluricanonical form. Then $\sigma(\psi) \in$ $H^{0}\left(X_{\varphi}, K_{X_{\varphi}}^{m}\right)$ is holomorphic with respect to the complex structure $J_{\varphi}$ if and only if

$$
\bar{\partial} \psi=\varphi\lrcorner \nabla \psi
$$

where $\bar{\partial}$ and $\nabla$ are operators on $X_{0}$.

Proof. The proof is similar to Lemma 10.2. Let $w$ be any holomorphic coordinate on $X_{\varphi}$. Then

$$
d w=\frac{\partial w}{\partial z} d z+\frac{\partial w}{\partial \bar{z}} d \bar{z}=\frac{\partial w}{\partial z}(d z+\varphi d \bar{z}) .
$$

Let $a=a(z, \bar{z})=\frac{\partial w}{\partial z}$ and let $\psi=f(z) d z^{m}$ be the local expression of $\psi$. Then locally we have

$$
\sigma(\psi)=f(z)(d z+\varphi d \bar{z})^{m}=\frac{f}{a^{m}} d w^{m} .
$$

Thus $\sigma(\psi)$ is holomorphic with respect to $J_{\varphi}$ if any only if

$$
\partial_{\bar{z}}\left(\frac{f}{a^{m}}\right)=\varphi \partial_{z}\left(\frac{f}{a^{m}}\right) .
$$

By using the fact that

$$
\frac{\partial a}{\partial \bar{z}}=\frac{\partial^{2} w}{\partial z \partial \bar{z}}=\partial_{z}\left(\frac{\partial w}{\partial \bar{z}}\right)=\partial_{z}\left(\varphi \frac{\partial w}{\partial z}\right)=\partial_{z}(a \varphi)
$$


we know that equation (10.15) is equivalent to

$$
\partial_{\bar{z}} f=m f \partial_{z} \varphi+\varphi \partial_{z} f
$$

Now we have

$$
\nabla \psi=\left(\partial_{z} f-f \partial_{z} \log \lambda^{m}\right) d z \otimes d z^{m}
$$

which implies

$$
\varphi\lrcorner \nabla \psi=\left(\partial_{z} f-f \partial_{z} \log \lambda^{m}\right) \varphi d \bar{z} \otimes d z^{m} .
$$

Since $\varphi$ is harmonic we know $\bar{\partial}^{*} \varphi=0$ which implies $\partial_{z}(\lambda \varphi)=0$. Namely

$$
\partial_{z} \varphi=-\varphi \partial_{z} \log \lambda .
$$

Now we insert formula (10.18) into formula (10.17) we get

$$
\varphi\lrcorner \nabla \psi=\left(\varphi \partial_{z} f+m f \partial_{z} \varphi\right) d \bar{z} \otimes d z^{m} .
$$

Since $\bar{\partial} \psi=\partial_{\bar{z}} f d \bar{z} \otimes d z^{m}$ the lemma follows from comparing formulas (10.19) and (10.16).

Now we construct canonical local sections of $E_{m}$. We fix a point $0 \in \mathcal{M}_{g}$ and a harmonic Beltrami differential $\varphi \in \mathbb{H}^{0,1}\left(X_{0}, T_{X_{0}}^{1,0}\right)$. We define the operator

$$
\rho=\rho_{\varphi}: A^{0}\left(X_{0}, K_{X_{0}}^{m}\right) \rightarrow A^{0}\left(X_{0}, K_{X_{0}}^{m}\right)
$$

by

$$
\left.\rho_{\varphi}(\psi)=\bar{\partial}^{*} G(\varphi\lrcorner \nabla \psi\right)
$$

for any $\psi \in A^{0}\left(X_{0}, K_{X_{0}}^{m}\right)$. Here $G$ is the Green operator.

Lemma 10.4. For any $\psi \in A^{0}\left(X_{0}, K_{X_{0}}^{m}\right)$ we have

(1) $\left.\bar{\partial}\left(\rho_{\varphi}(\psi)\right)=\varphi\right\lrcorner \nabla \psi$;

(2) $\mathbb{H}\left(\rho_{\varphi}(\psi)\right)=0$ where

$$
\mathbb{H}: A^{0}\left(X_{0}, K_{X_{0}}^{m}\right) \rightarrow H^{0}\left(X_{0}, K_{X_{0}}^{m}\right)
$$

is the harmonic projection.

Proof. The second claim follows from Hodge decomposition since

$$
\rho_{\varphi}(\psi) \in \operatorname{Im}\left(\bar{\partial}^{*}\right) .
$$

To prove the first claim we notice that

$$
\bar{\partial} G\left(\rho_{\varphi}(\psi)\right)=G \bar{\partial}\left(\rho_{\varphi}(\psi)\right)=0
$$


since $\rho_{\varphi}(\psi) \in A^{0,1}\left(X_{0}, K_{X_{0}}^{m}\right)$. Furthermore, since

$$
h^{1}\left(X_{0}, K_{X_{0}}^{m}\right)=h^{0}\left(X_{0}, K_{X_{0}}^{1-m}\right)=0
$$

as computed before, we know

$$
\mathbb{H}(\varphi\lrcorner \nabla \psi)=0 .
$$

Thus we have

$$
\begin{aligned}
\bar{\partial}\left(\rho_{\varphi}(\psi)\right) & \left.\left.=\overline{\partial \partial}^{*} G(\varphi\lrcorner \nabla \psi\right)=\left(\overline{\partial \partial}^{*}+\bar{\partial}^{*} \bar{\partial}\right) G(\varphi\lrcorner \nabla \psi\right) \\
& =\square G(\varphi\lrcorner \nabla \psi)=(I-\mathbb{H})(\varphi\lrcorner \nabla \psi)=\varphi\lrcorner \nabla \psi
\end{aligned}
$$

We finished the proof.

Now we fix a harmonic Beltrami differential $\varphi \in \mathbb{H}^{0,1}\left(X_{0}, T_{X_{0}}^{1,0}\right)$ and let $X_{t}=X_{t \varphi}$. For any $\psi \in H^{0}\left(X_{0}, K_{X_{0}}^{m}\right)$ which is holomorphic with respect to the complex structure $J_{0}$ on $X_{0}$, we let $\psi_{0}=\psi$ and let

$$
\psi_{n}=\left(\rho_{\varphi}\right)^{n}(\psi) \in A^{0}\left(X_{0}, K_{X_{0}}^{m}\right) .
$$

For any $t$ such that $|t|$ is small we define

$$
\psi(t)=\sum_{n=0}^{\infty} t^{n} \psi_{n} .
$$

THEOREM 10.1. We have

(1) The power series $\sum_{n=0}^{\infty} t^{n} \psi_{n}$ converges when $|t|$ small;

(2) For each $t$ we have

$$
\sigma_{t}(\psi(t)) \in H^{0}\left(X_{t}, K_{X_{t}}^{m}\right)
$$

is holomorphic with respect to the complex structure $J_{t}=J_{t \varphi}$.

Proof. The first claim follows from standard elliptic estimates which is similar to the proof of Proposition (10.1).

To prove the second claim, by Lemma 10.3 we only need to check

$$
\bar{\partial} \psi(t)=t \varphi\lrcorner \nabla \psi(t) .
$$

By looking at the coefficients of the term $t^{k}$ of both sides the equation, we know that the above equation is equivalent to

$$
\left\{\begin{array}{l}
\bar{\partial} \psi_{0}=0 \\
\left.\bar{\partial} \psi_{k}=\varphi\right\lrcorner \nabla \psi_{k-1} \quad \text { if } k \geq 1 .
\end{array}\right.
$$


However, the first equation is clear since $\psi_{0}=\psi$ is holomorphic with respect to $J_{0}$. The second equation follows from the construction of $\psi(t)$ and Lemma 10.4 .

REMARK 10.3. We call the family $\sigma_{t \varphi}(\psi(t))$ the canonical deformation of $\psi$ in the direction $\varphi$. By using its construction we can easily find its expansion on the central fiber $X_{0}$. The multi-variable case follows in the same manner.

In order to get the expansion of $\sigma_{t}\left(\psi_{t}\right)$ we consider the contraction $\left.\varphi\right\lrcorner \psi$. Let $\varphi$ be any Beltrami differential on $X_{0}$ and let $\psi \in A^{0}\left(X_{0}, K_{X_{0}}^{m}\right)$ be any pluricanonical form. With respect to any local holomorphic coordinate $z$ on $X_{0}$ we have $\varphi=\varphi(z) d \bar{z} \otimes \frac{\partial}{\partial z}$ and $\psi=f(z) d z^{m}$. We define

$$
\varphi\lrcorner \psi=m f \varphi d \bar{z} \otimes d z^{m-1} .
$$

Similar to formula (10.12) we have the expansion of the canonical local section of $E_{m}$.

COROLlary 10.2. The pluricanonical forms $\sigma_{t}(\psi(t))$ has expansion

$$
\left.\left.\sigma_{t}(\psi(t))=\psi_{0}+t\left(\bar{\partial}^{*} G(\varphi\lrcorner \nabla \psi_{0}\right)+\varphi\right\lrcorner \psi_{0}\right)+O\left(t^{2}\right)
$$

REMARK 10.4. We note that both terms $\left.\bar{\partial}^{*} G(\varphi\lrcorner \nabla \psi_{0}\right)$ and $\left.\varphi\right\lrcorner \psi_{0}$ can be viewed as section of the bundle $S^{m}\left(\Omega^{\mathbb{C}} X_{0}\right)$ where $\Omega^{\mathbb{C}} X_{0}$ is the complexified cotangent bundle and $S^{m}$ stands for symmetric product.

This method of canonically deforming the pluricanonical forms can be generalized to higher dimensional fibers with minor modifications.

\section{References}

[1] L. Ahlfors and L. Bers. Riemann's mapping theorem for variable metrics. Ann. of Math. (2), 72:385-404, 1960.

[2] S. Y. Cheng and S. T. Yau. On the existence of a complete Kähler metric on noncompact complex manifolds and the regularity of Fefferman's equation. Comm. Pure Appl. Math., 33(4):507-544, 1980.

[3] P. Deligne and D. Mumford. The irreducibility of the space of curves of given genus. Inst. Hautes Études Sci. Publ. Math., (36):75-109, 1969.

[4] F. Griffits and V. Šmid. Locally homogeneous complex manifolds. Uspehi Mat. Nauk, 26(5(161)):117-168, 1971. Translated from the English (Acta Math. 123 (1969), 253$302)$ by B. L. Žitnickiı̌.

[5] J. Harer and D. Zagier. The Euler characteristic of the moduli space of curves. Invent. Math., 85(3):457-485, 1986.

[6] L. Ji, K. Liu, X. Sun, and S.-T. Yau. Gauss-bonnet theorem on the moduli space of riemann surfaces. preprint, 2009.

[7] L. Keen. Collars on Riemann surfaces. In Discontinuous groups and Riemann surfaces (Proc. Conf., Univ. Maryland, College Park, Md., 1973), pages 263-268. Ann. of Math. Studies, No. 79. Princeton Univ. Press, Princeton, N.J., 1974. 
[8] M. Kuranishi. On deformations of compact complex structures. In Proc. Internat. Congr. Mathematicians (Stockholm, 1962), pages 357-359. Inst. Mittag-Leffler, Djursholm, 1963.

[9] K. Liu, X. Sun, and S.-T. Yau. Canonical metrics on the moduli space of Riemann surfaces. I. J. Differential Geom., 68(3):571-637, 2004.

[10] K. Liu, X. Sun, and S.-T. Yau. Canonical metrics on the moduli space of Riemann surfaces. II. J. Differential Geom., 69(1):163-216, 2005.

[11] K. Liu, X. Sun, and S.-T. Yau. Good geometry on the curve moduli. Publ. Res. Inst. Math. Sci., 44(2):699-724, 2008.

[12] K. Liu, X. Sun, and S.-T. Yau. New results on the geometry of the moduli space of Riemann surfaces. Sci. China Ser. A, 51(4):632-651, 2008.

[13] K. Liu, X. Sun, and S.-T. Yau. Good metrics on the moduli space of Riemann surface I. preprint, 2009.

[14] K. Liu, X. Sun, and S.-T. Yau. Good metrics on the moduli space of Riemann surface II. preprint, 2009.

[15] Z. Lu and X. Sun. Weil-Petersson geometry on moduli space of polarized Calabi-Yau manifolds. J. Inst. Math. Jussieu, 3(2):185-229, 2004.

[16] V. Markovic and D. Saric. The universal properties of teichmüller spaces. Surveys in Differential Geometry, 2009.

[17] H. Masur. Extension of the Weil-Petersson metric to the boundary of Teichmuller space. Duke Math. J., 43(3):623-635, 1976.

[18] C. T. McMullen. The moduli space of Riemann surfaces is Kähler hyperbolic. Ann. of Math. (2), 151(1):327-357, 2000.

[19] D. Mumford. Hirzebruch's proportionality theorem in the noncompact case. Invent. Math., 42:239-272, 1977.

[20] R. C. Penner. Perturbative series and the moduli space of Riemann surfaces. J. Differential Geom., 27(1):35-53, 1988.

[21] L. Saper. $L^{2}$-cohomology of the Weil-Petersson metric. In Mapping class groups and moduli spaces of Riemann surfaces (Göttingen, 1991/Seattle, WA, 1991), volume 150 of Contemp. Math., pages 345-360. Amer. Math. Soc., Providence, RI, 1993.

[22] W. Schmid. Variation of Hodge structure: the singularities of the period mapping. Invent. Math., 22:211-319, 1973.

[23] W.-X. Shi. Ricci flow and the uniformization on complete noncompact Kähler manifolds. J. Differential Geom., 45(1):94-220, 1997.

[24] Y. Shimizu and K. Ueno. Advances in moduli theory, volume 206 of Translations of Mathematical Monographs. American Mathematical Society, Providence, RI, 2002. Translated from the 1999 Japanese original, Iwanami Series in Modern Mathematics.

[25] S. Trapani. On the determinant of the bundle of meromorphic quadratic differentials on the Deligne-Mumford compactification of the moduli space of Riemann surfaces. Math. Ann., 293(4):681-705, 1992.

[26] S. A. Wolpert. Chern forms and the Riemann tensor for the moduli space of curves. Invent. Math., 85(1):119-145, 1986.

[27] S. A. Wolpert. Asymptotics of the spectrum and the Selberg zeta function on the space of Riemann surfaces. Comm. Math. Phys., 112(2):283-315, 1987.

[28] S. A. Wolpert. The hyperbolic metric and the geometry of the universal curve. $J$. Differential Geom., 31(2):417-472, 1990.

[29] S. A. Wolpert. Geometry of the Weil-Petersson completion of Teichmüller space. In Surveys in differential geometry, Vol. VIII (Boston, MA, 2002), Surv. Differ. Geom., VIII, pages 357-393. Int. Press, Somerville, MA, 2003.

[30] S. A. Wolpert. Understanding weil-petersson curvature. arXiv:0809.3699, 2008.

[31] S. A. Wolpert. Extension of the Weil-Petersson connection. Duke Math. J., 146(2):281-303, 2009. 
[32] S. A. Wolpert. The Weil-Petersson metric geometry. In Handbook of Teichmüller theory. Vol. II, volume 13 of IRMA Lect. Math. Theor. Phys., pages 47-64. Eur. Math. Soc., Zürich, 2009.

[33] S. Yeung. Geometry of domains with the uniform squeezing property. arXiv:0906.4647, 2009.

Department of Mathematics, University of California at Los Angeles, Los Angeles, CA 90095-1555, USA, Center of Mathematical Sciences, Zhejiang University, HANGZHOU, ChinA

E-mail address: liu@math.ucla.edu, liu@cms.zju.edu.cn

Department of Mathematics, Lehigh University, Bethlehem, PA 18015, USA

E-mail address: xis205@lehigh.edu

Department of Mathematics, Harvard University Cambridge, MA 02138, USA

E-mail address: yau@math.harvard.edu 
